\title{
Radio continuum morphology of southern Seyfert galaxies ${ }^{\star}$
}

\author{
R. Morganti ${ }^{1}$, Z.I. Tsvetanov ${ }^{2}$, J. Gallimore ${ }^{3, \star \star}$, and M.G. Allen ${ }^{4}$ \\ 1 Istituto di Radioastronomia, CNR, Via Gobetti 101, 40129 Bologna, Italy \\ e-mail: rmorgant@ira.bo.cnr.it \\ 2 Department of Physics and Astronomy, Johns Hopkins University, Baltimore, MD 21218, U.S.A. \\ 3 NRAO, 520 Edgemont Rd., Charlottesville, VA 22903, U.S.A. \\ 4 Mount Stromlo and Siding Spring Observatories, Private Bag, Weston Creek Post Office, ACT 2611, Australia
}

Received October 19, 1998; accepted April 16, 1999

\begin{abstract}
We present radio observations for 29 southern Seyfert galaxies selected from a volume limited sample with $c z<3600 \mathrm{~km} \mathrm{~s}^{-1}$, and declination $\delta<0^{\circ}$. Objects with declination $-30^{\circ}<\delta<0^{\circ}$ were observed with the Very Large Array (VLA) at $6 \mathrm{~cm}(4.9 \mathrm{GHz})$ and objects with $\delta<-30^{\circ}$ were observed with the Australia Telescope Compact Array (ATCA) at $3.5 \mathrm{~cm}(8.6 \mathrm{GHz})$. Both the VLA and the ATCA observations have a resolution of $\sim 1^{\prime \prime}$. These new observations cover more than $50 \%$ of the southern sample with all but two of the 29 objects detected above our limit of $0.15 \mathrm{mJy}$. Combining these data with data available from the literature gives almost $85 \%$ coverage of the southern sample and further expands the radio observations of a distance limited sample by $22 \%$.

Collecting additional sources from the literature, consisting of known Seyferts with $c z<4600 \mathrm{~km} \mathrm{~s}^{-1}$, we find that $38 \%$ of the sources are unresolved at arcsecond resolution, and $34 \%$ have linear radio structure. Our results expand upon and are consistent with earlier studies. We confirm a correlation between the size of the radio structure and the radio luminosity. A comparison between Seyfert types 1 and 2 finds that type 2 s tend to have a larger linear size. There is no statistically significant difference in radio power between types 1 and 2, although all the most powerful objects appear to be Seyfert 2's. No significant difference has been found in the spectral indices.
\end{abstract}

Key words: radio continuum: galaxies — galaxies: Seyfert

Send offprint requests to: $\mathrm{R}$. Morganti

* Based on observations done with the Australia Telescope Compact Array (ATCA) and the Very Large Array (VLA).

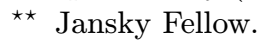

\section{Introduction}

Radio emission from Seyfert galaxies traces energetic, mechanical processes occurring in the active nucleus. The radio continuum spectra of Seyferts are almost invariably steep, consistent with optically-thin synchrotron emission. In those sources where the radio continuum has been resolved, the morphology is commonly linear, interpreted to trace a stream of ejected plasma, or a jet, originating from the central engine. For example, most well-resolved Seyfert radio sources are double or triple sources straddling the active nucleus. The radio emission of the nearest and brightest Seyferts resolves into tightly collimated structures originating from the active nucleus, consistent with a jet interpretation (e.g., NGC 1068: Gallimore et al. 1996; Muxlow et al. 1996; Wilson \& Ulvestad 1983; NGC 4151: Pedlar et al. 1993; Mrk 3: Kukula et al. 1993). There is evidence that Seyfert radio jets impact the dense gas in the near-nucleus environment and affect the gas distribution, morphology, and ionization of the narrow-line region (NLR). The orientation of the radio jet is also the only straightforward measure of the symmetry axis of the active nucleus, useful for testing orientation-based unifying schemes for AGNs.

Early studies searching for differences between Seyfert 1's and 2's (de Bruyn \& Wilson 1978; Meurs \& Wilson; Ulvestad \& Wilson 1984a; Ulvestad \& Wilson 1984b) concluded that Seyfert 2's have stronger and larger radio sources than Seyfert 1's, however, these studies were biased by optical selection: weaker Seyfert 2 galaxies were omitted from the samples. There was found to be little or no difference between Seyfert 1 and 2 radio sources in follow-up studies which properly considered relatively unbiased, volume-limited samples (Edelson 1987; Ulvestad \& Wilson 1989; Giuricin et al. 1990). More specifically, there is no statistically significant difference in the distribution of radio luminosity, and only a marginal difference in the distribution of radio source 
sizes, with Seyfert 2's tending to be slightly larger than Seyfert 1's (although only at $<90 \%$ significance, Wilson 1991). In contrast, unifying schemes predict that the radio jets in Seyfert 2's should appear larger in projection, since the unifying model orients the collimating disk more nearly edge-on in narrow-line AGNs. It is not simple to reconcile the statistics of radio sources with the unified scheme hypothesis.

While there is no tendency for the radio sources to orient in any preferred direction with respect to the plane of the host galaxy (Ulvestad \& Wilson 1984a), the radio sources are commonly elongated in the same direction as the NLR (Haniff et al. 1988; Pogge 1989; Wilson et al. 1988), i.e. the inner part of the optical emission regions (extending up to few kpc). In some Seyfert $2 \mathrm{~s}$ the NLR has a conical or bi-conical shape with the nucleus at the apex (e.g. Pogge 1989, 1997). Wilson \& Tsvetanov (1994) showed that the radio axis is invariably co-aligned with the ionization cone axis for the 11 ionization cones known at that time.

The alignment of the radio jet and NLR raises a question of energetics - does the radio jet ionize and heat the NLR significantly compared to ionizing radiation from the AGN? In several well-studied cases, there is a detailed morphological association between the NLR and the radio emission (e.g. Whittle et al. 1988; Whittle 1989; Capetti et al. 1996; Gallimore et al. 1996). Currently, the numbers of such well-studied cases is too small to address questions of interaction and energetics in a statistically complete and meaningful sense.

To this end, Tsvetanov et al. (in preparation) have recently assembled a volume-limited sample (cz< $3600 \mathrm{~km} \mathrm{~s}^{-1}$ ) of well-classified Seyfert galaxies. The main advantage of this sample is that all of the sources have been extensively observed in the optical with narrow-band imaging done with the ESO NTT, $3.6 \mathrm{~m}$ and $2.2 \mathrm{~m}$ telescopes. This survey provides high-quality emission-line maps (in [O III $] \lambda 5007$ and $\mathrm{H} \alpha+[\mathrm{N} \mathrm{II}]$ ) with a typical resolution of $\sim 1^{\prime \prime}$. The sample includes objects with and without known extended emission line regions, providing detailed information about the extent, morphology, and degree of ionization (obtained from the [O III $] \lambda 5007 / \mathrm{H} \alpha+[\mathrm{N} \mathrm{II}]$ ratio) of the emission-line regions. It is therefore important to obtain for such a sample detailed radio images in order to perform a detailed comparison of the radio and optical morphology on arcsecond (hundred-pc) scales.

Here we present a radio imaging survey of the Tsvetanov et al. sample, made using the Very Large Array $(\mathrm{VLA})^{1}$ and the Australia Telescope Compact

\footnotetext{
1 The National Radio Astronomy Observatory is operated by Associated Universities, Inc., under contract with National Science Foundation.
}

Array (ATCA) ${ }^{2}$ aperture synthesis telescopes. Observing configurations and frequencies were chosen to match the $\sim 1^{\prime \prime}$ resolution of the optical narrow-band observations. We discuss the radio properties and statistics for the 29 surveyed sources, and a more detailed comparison of the radio and optical properties will be presented in a forthcoming paper.

\section{Observations}

\subsection{Sample}

The sample includes 51 well classified (from their optical spectra) Seyfert galaxies south of declination $0^{\circ}$ and with redshift $c z<3600 \mathrm{~km} \mathrm{~s}^{-1}$. More details about selection and optical observations of this sample are presented in Tsvetanov et al. (in preparation), Tsvetanov et al. (1998).

The 29 sources listed in Table 1 were observed with either the VLA or ATCA, depending on their declination. One source, NGC 3393, was observed with both arrays. The array configurations and frequencies were chosen to obtain a resolution of $\sim 1^{\prime \prime}$ in order to match the resolution of the optical data. In particular, the VLA was used in its $B$ and $B_{\mathrm{N}} A$ configurations, providing a resolution of $\sim 1^{\prime \prime}$ at $6 \mathrm{~cm}(4.9 \mathrm{GHz})$. To obtain similar resolution with the ATCA required an observing frequency of $3.5 \mathrm{~cm}(8.6 \mathrm{GHz})$ with the $6 \mathrm{~km}$ configuration. Owing to missing short baselines in either array, our data are more sensitive to compact emission from the circumnuclear region but less sensitive to extended, diffuse emission. In addition, faint, diffuse emission from active spirals tends to have very steeply falling radio spectra with increasing frequency; at observing wavelengths of 3.5 and $6 \mathrm{~cm}$ any diffuse emission will probably have dropped below our surface brightness detection limit.

Many of those sources lying north of $\delta \sim-30^{\circ}$ have been observed in previous studies. The data available in the literature will be included in our discussion in Sect. 4 .

Thoughout the paper we adopt a Hubble constant $H_{0}=75 \mathrm{~km} \mathrm{~s}^{-1} \mathrm{Mpc}^{-1}$.

\subsection{VLA observations}

VLA snapshot observations were obtained for eight of the survey Seyferts in the declination range $-30^{\circ}<\delta<0^{\circ}$. The VLA observations are summarized in Table 1 . The observations were carried out using the standard $6 \mathrm{~cm}$ $(4.9 \mathrm{GHz})$ continuum mode, that is, with two $50 \mathrm{MHz}-$ wide channels at bandwidth-separated frequencies (4.835 $\& 4.885 \mathrm{GHz}$ ). Three sources were observed using the

\footnotetext{
${ }^{2}$ Operated by the CSIRO Australia Telescope National Facility.
} 
Table 1. VLA observations $(6 \mathrm{~cm})$

\begin{tabular}{|c|c|c|c|c|c|c|c|c|}
\hline \multirow[t]{2}{*}{ Object } & \multirow[t]{2}{*}{$\alpha_{2000}$} & \multirow[t]{2}{*}{$\delta_{2000}$} & \multicolumn{3}{|c|}{ Resolution } & \multirow{2}{*}{$\begin{array}{c}\mathrm{rms} \\
\text { mJy beam }^{-1}\end{array}$} & \multirow{2}{*}{$\begin{array}{c}\text { Peak } \\
\text { mJy beam }^{-1}\end{array}$} & \multirow[t]{2}{*}{$\overline{\text { Array }}$} \\
\hline & & & $\operatorname{arcsec}$ & $\operatorname{arcsec}$ & deg & & & \\
\hline NGC 1097 & 024619.1 & -301628 & 2.1 & 1.3 & -59 & 0.051 & 3.78 & $B_{\mathrm{N}} A$ \\
\hline NGC 1320 & 032448.7 & -030233 & 2.5 & 1.4 & -38 & 0.081 & 1.98 & $B$ \\
\hline NGC 3393 & 104824.0 & -250940 & 2.2 & 1.3 & -3 & 0.049 & 15.80 & $B_{\mathrm{N}} A$ \\
\hline NGC 3660 & 112332.2 & -083930 & 2.1 & 1.4 & -1 & 0.049 & 0.49 & $B$ \\
\hline NGC 4968 & 130706.0 & -234043 & 2.3 & 1.4 & -4 & 0.046 & 13.06 & $B_{\mathrm{N}} A$ \\
\hline NGC 5427 & 140325.9 & -060150 & 2.1 & 1.4 & -17 & 0.048 & 2.49 & $B$ \\
\hline NGC 7172 & 220202.1 & -315212 & 3.6 & 1.0 & 36 & 0.053 & 3.62 & $B_{\mathrm{N}} A$ \\
\hline NGC 7314 & 223546.0 & -260302 & 3.0 & 1.2 & 31 & 0.034 & 2.74 & $B_{\mathrm{N}} A$ \\
\hline
\end{tabular}

$B$-array (8 Apr. 1993); the remaining five were observed using the $B_{\mathrm{N}}{ }^{3}$ array (1 Feb. 1993).

Data reduction followed standard procedures using the NRAO software package AIPS. The calibration sequence includes: (1) calibrating the flux scale against observations of the flux standards 3C 48 \& 3C 286, adopting the standard scaling of Baars et al. (1977) and adjusting for calibrator variability; (2) bootstrapping this flux scale to observations of the phase calibrators; (3) performing station-based phase and amplitude calibrations on the phase calibrators; and (4) applying the phase calibrator solutions to the target sources. Four iterations of phaseonly self-calibration were then applied to each source. Solution intervals were typically $5-10$ minutes for each iteration of self-calibration.

\subsection{ATCA observations}

The ATCA observing parameters are summarized in Table 2. Twenty-two objects in the most southern part of this sample $\left(\delta<-30^{\circ}\right)$ were observed at $3.5 \mathrm{~cm}$ $(8.6 \mathrm{GHz})$ with the ATCA in $6 \mathrm{~km}$ configuration. The only overlap with the VLA observations is NGC 3393.

The observations occurred during July, 1995. Data were taken simultaneously at 8.256 and $8.896 \mathrm{GHz}$, each through a bandwidth of $128 \mathrm{MHz}$. These dual-frequency observations improved the coverage of the $(u, v)$-plane and the sensitivity. Use of dual-frequency synthesis substantially improves the image quality generated by data from sparse arrays.

We used the MIRIAD package (Sault et al. 1995) for data reduction. The flux density scale was calibrated against observations of PKS 1934-638, assumed to be $2.84 \mathrm{Jy}$ at $8.6 \mathrm{GHz}$ according to the latest analysis by Reynolds (1996). Each source was observed in 8 scans with a duration of roughly 20 min each. The scans were spread

\footnotetext{
3 The $B_{\mathrm{N}}$ configuration comprises the east-west portion of the $B$-array and extended northern arm; this configuration provides roughly $B$-array resolution for southern sources.
}

throughout a 12 hour observing period in order to optimize the $(u, v)$ coverage within the available integration time. Sources with particularly weak or interesting structure were repeated in order to improve the signal-to-noise and image quality.

\section{Results}

Table 3 summarizes our measurements for the 29 observed Seyferts. The listed radio morphologies follow the convention of Ulvestad \& Wilson (1989). The angular and linear size of the radio emission, measured for those Seyferts with well-resolved radio structure, were measured to the outermost significant surface brightness contour; upper limits are given for unresolved Seyferts. The radio maps of the resolved objects are presented in Figs. 1 to 4 for the objects observed with the VLA and in Figs. 5 to 15 for the objects observed with ATCA. To emphasize ring or shell structures that can be lost on contour maps, we present all maps as contours on top of greyscale.

Most of the resolved sources bear a linear radio structure as is commonly observed in Seyfert nuclei. In NGC 1097 and NGC 1365 we resolve a central, unresolved radio source, apparently related to the AGN, and a ring associated with star forming regions (compare with Hummel et al. 1987 and Sandqvist et al. 1995, respectively). NGC 7582 displays diffuse radio structure. Only two objects of the observed (IC 5201 and NGC 7590) were undetected; both are part of the group observed at $3 \mathrm{~cm}$ with ATCA.

Previous observations for 9 of the 29 objects have been reported in the literature by various authors. Where possible we have used these data in combination with our new observations to estimate a radio spectral index, $\alpha$, defined as $S \propto \nu^{\alpha}$. We caution that there are inestimable uncertainties in the spectral indices for extended sources owing to differences in the $(u, v)$ coverage between our observations and those in the literature. Nevertheless, the indices are accurate for unresolved sources and otherwise provide a sense of the spectral shape for the extended sources. 
Table 2. ATCA observations $(3.5 \mathrm{~cm})$

\begin{tabular}{|c|c|c|c|c|c|c|c|}
\hline \multirow[t]{2}{*}{ Object } & \multirow[t]{2}{*}{$\alpha_{2000}$} & \multirow[t]{2}{*}{$\delta_{2000}$} & \multicolumn{3}{|c|}{ Resolution } & \multirow{2}{*}{$\begin{array}{r}\mathrm{rms} \\
\mathrm{mJy} \text { beam }^{-1}\end{array}$} & \multirow{2}{*}{$\begin{array}{r}\text { Peak } \\
\text { mJy beam }^{-1}\end{array}$} \\
\hline & & & $\operatorname{arcsec}$ & $\operatorname{arcsec}$ & degrees & & \\
\hline TOL 0109-383 & 011127.8 & -380458 & 2.58 & 0.83 & 46.0 & 0.14 & 10.43 \\
\hline NGC 1365 & 033335.5 & -360822 & 1.61 & 0.77 & 5.4 & 0.11 & 4.06 \\
\hline NGC 1386 & 033646.3 & -355958 & 1.68 & 0.75 & -0.6 & 0.13 & 9.88 \\
\hline NGC 1566 & 042000.3 & -545618 & 1.29 & 0.75 & 17.5 & 0.17 & 5.65 \\
\hline ESO 362-G18 & 051935.5 & -323930 & 1.89 & 0.75 & -5.7 & 0.14 & 2.40 \\
\hline IC 2560 & $1016 \quad 19.2$ & -333353 & 1.76 & 0.94 & -19.3 & 0.26 & 3.86 \\
\hline NGC 3281 & 103152.1 & -345115 & 1.67 & 0.80 & -16.3 & 0.20 & 16.06 \\
\hline NGC 3393 & 104824.0 & -250940 & 2.29 & 0.76 & -0.5 & 0.17 & 7.33 \\
\hline NGC 3783 & 113901.7 & -374419 & 1.59 & 0.74 & -10.5 & 0.24 & 8.15 \\
\hline NGC 4507 & 123537.0 & -395431 & 1.45 & 0.83 & 25.0 & 0.16 & 3.18 \\
\hline TOL 1238-364 & 124052.8 & -364521 & 2.42 & 0.88 & 50.0 & 0.16 & 10.29 \\
\hline MCG-6-30-15 & 133553.7 & -341745 & 1.66 & 0.79 & 1.2 & 0.16 & 0.62 \\
\hline NGC 5643 & 143241.3 & -441024 & 1.50 & 0.71 & -1.7 & 0.10 & 4.36 \\
\hline ESO 137-G34 & 163514.2 & -580441 & 1.32 & 0.70 & 32.6 & 0.12 & 2.02 \\
\hline ESO 138-G01 & 165120.5 & -591411 & 1.42 & 0.71 & 45.9 & 0.14 & 4.34 \\
\hline NGC 6221 & 165246.6 & -591259 & 1.29 & 0.72 & 27.6 & 0.16 & 2.96 \\
\hline NGC 6300 & 171659.2 & -624911 & 1.48 & 0.72 & 28.3 & 0.14 & 2.03 \\
\hline IC 5063 & 205202.8 & -570414 & 1.05 & 0.81 & 51.8 & 0.15 & 160.01 \\
\hline IC 5201 & 222057.3 & -460204 & 1.32 & 0.82 & 3.0 & 0.15 & $<0.45$ \\
\hline NGC 7496 & 230946.9 & -432542 & 1.94 & 0.67 & 16.1 & 0.12 & 4.83 \\
\hline NGC 7582 & 231823.1 & -422211 & 1.29 & 0.78 & 2.7 & 0.10 & 6.22 \\
\hline NGC 7590 & 231855.0 & -421416 & 1.39 & 0.88 & 7.2 & 0.30 & $<0.90$ \\
\hline
\end{tabular}

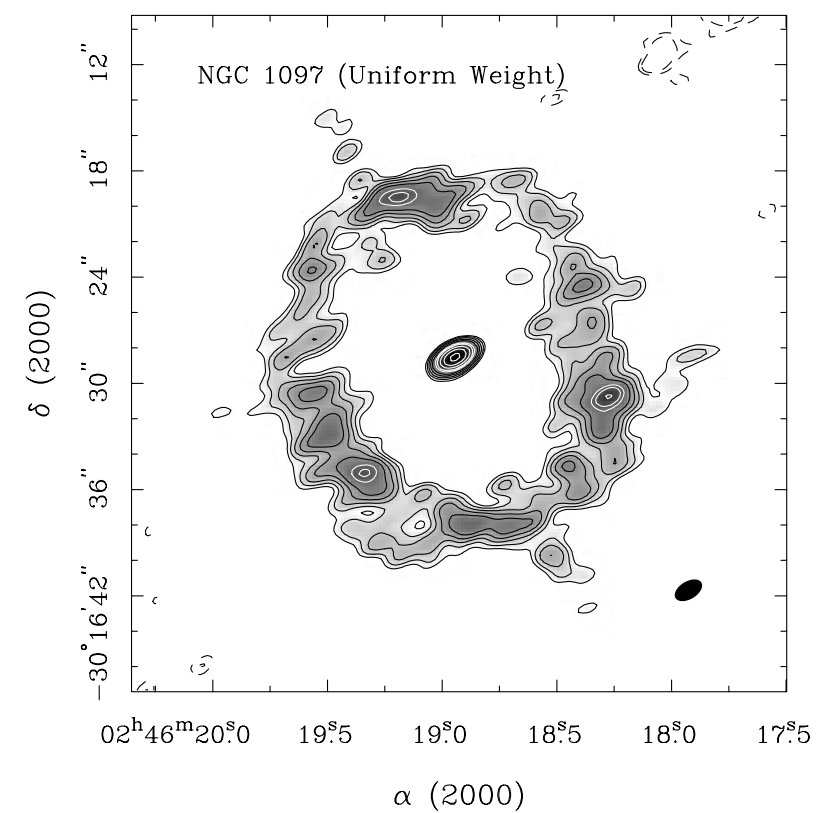

Fig. 1. NGC 1097 (uniform weighting) Contours: $1.0 \mathrm{mJy}$ beam $^{-1} \times-0.16,-0.22,0.16,0.22,0.31,0.44,0.62,0.97$, $1.21,1.70,2.38,3.33$

In the next section we discuss each source individually and also summarize relevant radio and optical observations available in the literature. Where possible, the flux at $20 \mathrm{~cm}(1.4 \mathrm{GHz})$, and the Parkes-Tidbinbilla interferometer (PTI) flux measurements at $13 \mathrm{~cm}(2.3 \mathrm{GHz})$ taken from literature, are listed. The PTI observations have an effective resolution of $\sim 0$ "! 1 , much higher that the data presented in this paper. The main information we can derive from PTI observations is whether a compact radio structure $(<0$.' 1$)$ is present. Where possible we compute spectral indices between 20 and $6 \mathrm{~cm}\left(\alpha_{6}^{20}\right)$ and between 6 and $3 \mathrm{~cm}\left(\alpha_{3}^{6}\right)$, and compare with previously reported values.

Our snapshot observations are insensitive to structures larger than $15-18$ arcsec, or around $4 \mathrm{kpc}$ at the redshift limit of this survey. The observations therefore resolve out diffuse emission from galaxy disks, but we remain sensitive to radio emission from compact star-forming regions. An interesting result of this survey is that star-forming rings are not common. Only NGC 1097 and NGC 1365 display starburst rings, similar to the one observed in NGC 1068, for example, even though our observations would have detected and resolved such rings out to the redshift limit of the survey. Otherwise the radio structures resolved by this survey resemble the classic linear radio structures commonly observed in Seyfert nuclei, and we assume that these structures are jets powered by the AGN. Of course, we are unable to distinguish jets and compact nuclear starbursts in unresolved sources, corresponding to linear scales $\lesssim 500$ pc at the redshift limit of the survey.

\subsection{Notes on individual sources}

TOL 0109-383 (NGC 424): This object has been observed by Ulvestad \& Wilson (1989) at 20 and $6 \mathrm{~cm}$. With 
Table 3. The radio parameters from VLA \& ATCA observations

\begin{tabular}{|c|c|c|c|c|c|c|c|}
\hline Object & $\begin{array}{r}c z \\
\mathrm{~km} \mathrm{~s}^{-1}\end{array}$ & $\begin{array}{r}\text { Type } \\
\text { Sey }\end{array}$ & $\begin{array}{r}S_{6 \mathrm{~cm}} \\
\mathrm{mJy}\end{array}$ & $\begin{array}{c}\log P_{6 \mathrm{~cm}} \\
W \mathrm{~Hz}^{-1}\end{array}$ & $\begin{array}{l}\text { Radio } \\
\text { Morp. }\end{array}$ & $\begin{array}{r}\text { LAS } \\
\operatorname{arcsec}\end{array}$ & $\begin{array}{r}\mathrm{LLS} \\
\mathrm{kpc}\end{array}$ \\
\hline NGC 1097 & 1276 & $1^{*}$ & 62.3 & 21.33 & $\mathrm{U}+\mathrm{R}$ & 16.0 & 1.32 \\
\hline NGC 1097 nucleus & $\ldots$ & $1^{*}$ & 3.8 & 20.12 & $\mathrm{U}$ & $<0.2$ & $<0.02$ \\
\hline NGC 1097 ring & $\ldots$ & $1^{*}$ & 58.5 & 21.30 & $\mathrm{R}$ & 16.0 & 1.32 \\
\hline NGC 1320 & 2716 & 2 & 2.0 & 20.51 & $\mathrm{U}$ & $<0.8$ & $<0.14$ \\
\hline NGC 3393 & 3710 & 2 & 23.1 & 21.83 & $\mathrm{~L}$ & 2.7 & 0.65 \\
\hline NGC 3393 SW peak & $\ldots$ & 2 & 16.0 & 21.67 & $\mathrm{U}$ & $<0.4$ & $<0.10$ \\
\hline NGC 3393 NE peak & $\ldots$ & 2 & 5.0 & 21.16 & $\mathrm{~S}$ & 1.3 & 0.31 \\
\hline NGC 3660 & 3300 & 2 & 0.5 & 20.06 & $\mathrm{U}$ & $<1.6$ & $<0.34$ \\
\hline NGC 4968 & 3007 & 2 & 13.1 & 21.40 & $\mathrm{U}$ & $<0.7$ & $<0.14$ \\
\hline NGC 5427 & 2565 & 2 & 2.5 & 20.54 & $\mathrm{U}$ & $<0.5$ & $<0.08$ \\
\hline NGC 7172 & 2527 & 2 & 11.7 & 21.20 & $\mathrm{~L}$ & 6.4 & 1.05 \\
\hline NGC 7314 & 1430 & 1.9 & 2.7 & 20.07 & $\mathrm{U}$ & $<0.5$ & $<0.05$ \\
\hline \multirow[t]{2}{*}{ Object } & $c z$ & Type & $S_{3 \mathrm{~cm}}$ & $\log P_{3 \mathrm{~cm}}$ & Radio & LAS & $\overline{\mathrm{LLS}}$ \\
\hline & $\mathrm{km} \mathrm{s}^{-1}$ & Sey & $\mathrm{mJy}$ & $W \mathrm{~Hz}^{-1}$ & Morp & $\operatorname{arcsec}$ & $\mathrm{kpc}$ \\
\hline TOL 0109-383 & 3496 & 2 & 13.5 & 21.54 & S? & 3 & 0.68 \\
\hline NGC 1365 & 1662 & 2 & 98.2 & 21.76 & $\mathrm{R}+\mathrm{L}$ & 20 & 2.15 \\
\hline NGC 1365 "jet" & $\ldots$ & $\ldots$ & $\ldots$ & $\ldots$ & $\mathrm{L}$ & 5 & 0.54 \\
\hline NGC 1386 & 924 & 2 & 12.7 & 20.36 & $\mathrm{U}$ & $<1.5$ & $<0.09$ \\
\hline NGC 1566 & 1496 & 1 & 8.0 & 20.58 & $\mathrm{~S} ?$ & 3 & 0.29 \\
\hline ESO $362-G 18$ & 3777 & 1 & 3.0 & 20.95 & $\mathrm{U}$ & $<1.5$ & $<0.37$ \\
\hline IC 2560 & 2873 & 2 & 6.2 & 21.03 & $\mathrm{U}$ & $<1.5$ & $<0.28$ \\
\hline NGC 3281 & 3460 & 2 & 18.1 & 21.66 & $\mathrm{~S}$ & 2.0 & 0.45 \\
\hline NGC 3393 & 3710 & 2 & 13.3 & 21.59 & $\mathrm{~L}$ & 3.0 & 0.72 \\
\hline NGC 3393 SW peak & $\ldots$ & $\ldots$ & 9.3 & 21.43 & $\ldots$ & $\ldots$ & $\ldots$ \\
\hline NGC 3393 NE peak & $\ldots$ & $\ldots$ & 3.3 & 20.98 & $\ldots$ & $\ldots$ & $\ldots$ \\
\hline NGC 3783 & 3208 & 1 & 10.03 & 21.34 & $\mathrm{U}$ & $<1.5$ & $<0.31$ \\
\hline NGC 4507 & 3957 & 2 & 5.57 & 21.27 & $\mathrm{~S}$ & 3 & 0.77 \\
\hline TOL 1238-364 & 3292 & 2 & 10.58 & 21.39 & $\mathrm{U}$ & $<2.0$ & $<0.43$ \\
\hline MCG-6-30-15 & 2248 & 1 & 1.12 & 20.08 & $\mathrm{U}$ & $<1.5$ & $<0.22$ \\
\hline NGC 5643 & 1199 & 2 & 5.68 & 20.24 & $\mathrm{U}$ & $<1.5$ & $<0.12$ \\
\hline ESO 137-G34 & 2620 & 2 & 9.84 & 21.16 & $\mathrm{~L}$ & 4.0 & 0.68 \\
\hline ESO 138-G01 & 2740 & 2 & 7.79 & 21.09 & $\mathrm{~S}$ & 3.0 & 0.53 \\
\hline NGC 6221 & 1482 & $2 ?$ & 14.91 & 20.84 & $\mathrm{~L}$ & 5.0 & 0.48 \\
\hline NGC 6300 & 1110 & 2 & 2.70 & 19.85 & $\mathrm{~S}$ & 3.5 & 0.25 \\
\hline IC 5063 & 3402 & 2 & 229.09 & 22.75 & $\mathrm{~L}$ & 4.2 & 0.92 \\
\hline IC 5201 & 915 & 2 & $<0.45$ & $<18.90$ & $\ldots$ & $\ldots$ & $<0.08$ \\
\hline NGC 7496 & 1649 & 2 & 6.52 & 20.57 & $\mathrm{U}$ & $<1.5$ & $<0.16$ \\
\hline NGC 7582 & 1575 & 2 & 49.29 & 21.41 & $\mathrm{D}$ & 6 & 0.61 \\
\hline NGC 7590 & 1596 & 2 & $<0.9$ & $<19.69$ & $\ldots$ & $\ldots$ & $<0.14$ \\
\hline
\end{tabular}

Radio Morphology: $\mathrm{L}=$ linear; $\mathrm{R}=$ ring; $\mathrm{D}=$ diffuse; $\mathrm{A}=$ ambiguous; $\mathrm{S}=$ slightly resolved; $\mathrm{U}=$ unresolved.

* NGC 1097 is a LINER that periodically shows Sy1-like broad lines (Storchi-Bergmann et al. 1993).

$\sim 1^{\prime \prime}$ resolution they found a slightly extended source with flux of $22.3 \mathrm{mJy}$ at $20 \mathrm{~cm}$, and $14.9 \mathrm{mJy}$ at $6 \mathrm{~cm}$. In our data we also find a structure that is slightly extended to the east (Fig. 5), although the very elongated beam of our observations makes this very uncertain. Ulvestad \& Wilson (1989) found that the dominant component has a flat spectrum with a spectral index between 20 and $6 \mathrm{~cm}$ of $\alpha_{20}^{6}=-0.17$. Our $3 \mathrm{~cm}$ measurement shows that the spectrum remains flat between 6 and $3 \mathrm{~cm}, \alpha_{6}^{3}=-0.21$.

NGC 1097: Originally classified as a LINER by the optical emission-line spectrum, the recent appearance of broad Balmer-line emission and a featureless blue continuum implies that it has a Seyfert 1 nucleus (Storchi-Bergmann et al. 1993). The radio structure (Fig. 1) comprises an unresolved point source and the well-known star-forming 


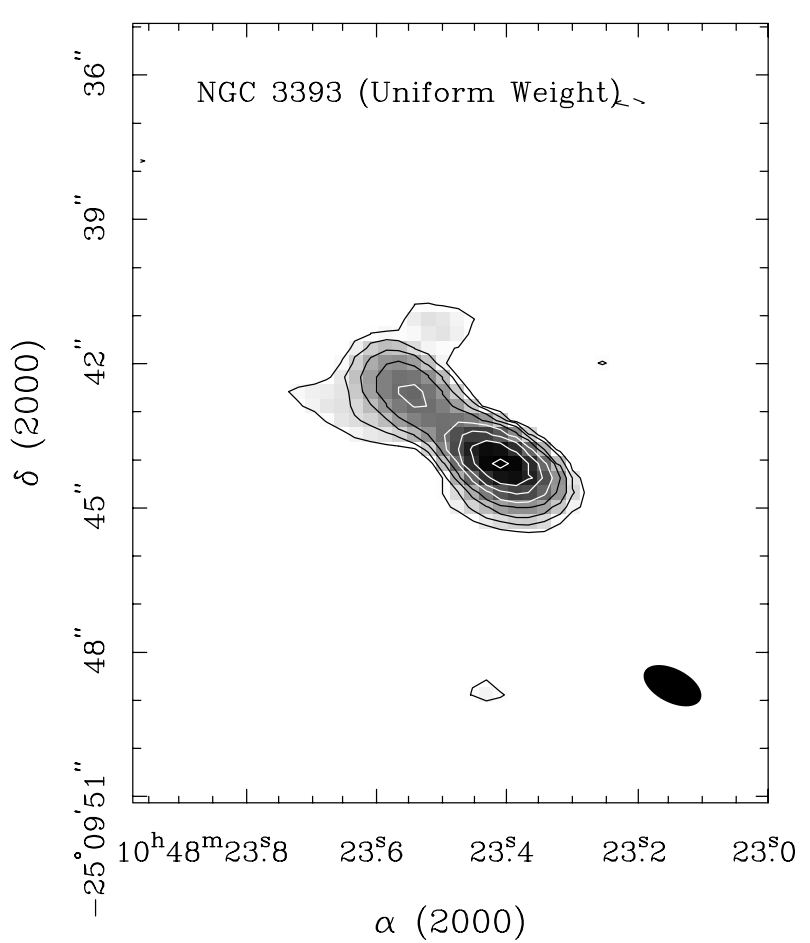

Fig. 2. NGC 3393. Contours: $1.0 \mathrm{mJy}$ beam $^{-1} \times-0.20,-0.36$, $0.20,0.36,0.66,1.20,2.19,4.02,7.36,13.5$

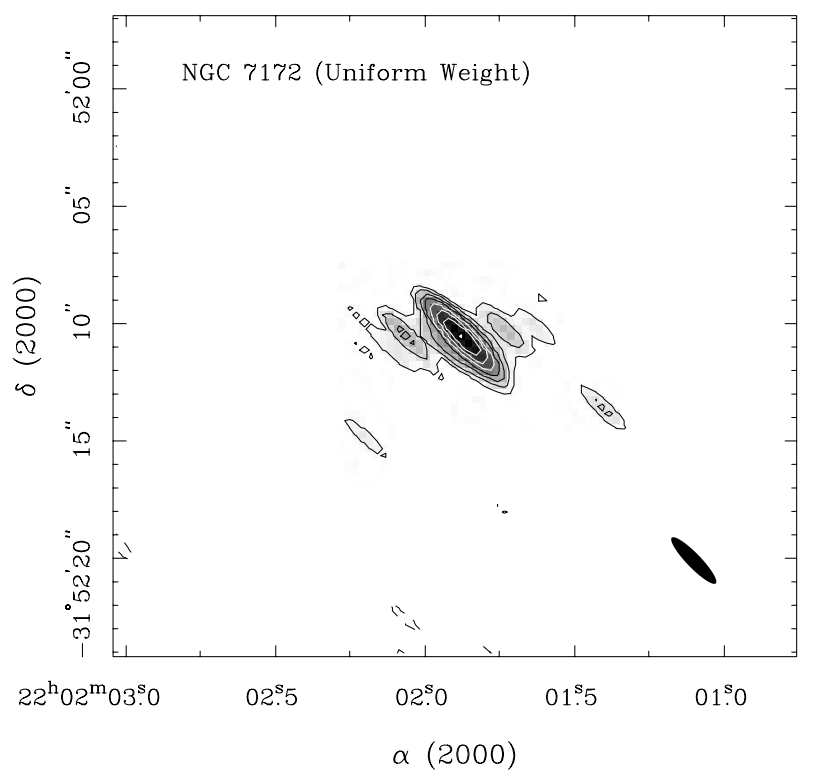

Fig. 3. NGC 7172. Contours: $1.0 \mathrm{mJy}^{\text {beam }}{ }^{-1} \times-0.16,-0.25$, $0.16,0.25,0.38,0.57,0.87,1.32,2.00,3.04$

ring. A detailed radio study of this object was performed by Hummel et al. (1987) and shows an overall steep spectrum ( $\alpha$ between -0.6 and -0.8$)$ and an inverted spectrum $(\alpha=1.0)$ for the nuclear component. The flux of the nucleus measured from our data is very similar to that found by Hummel et al. (1987). An upper limit to the core flux $\left(S_{13 \mathrm{~cm}}<5 \mathrm{mJy}\right)$ has been obtained from PTI observations by Sadler et al. (1995, hereafter S95).

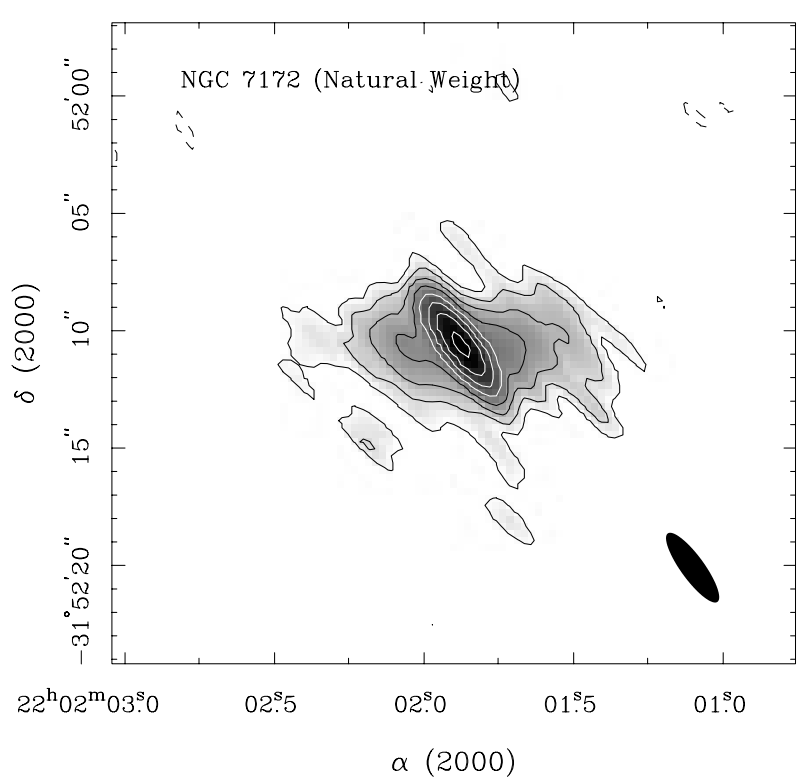

Fig. 4. NGC 7172. Contours: $1.0 \mathrm{mJy}^{\text {beam }}{ }^{-1} \times-0.16,-0.25$, $0.16,0.25,0.38,0.58,0.89,1.38,2.12,3.26$

NGC 1365: This is a well known southern barred galaxy. It was observed in the radio continuum $(20,6$ and $2 \mathrm{~cm})$ by Sandqvist et al. (1995, hereafter SJL95). As in SJL95, our map (Fig. 6) shows a ring of emission with angular dimensions $8^{\prime \prime} \times 20^{\prime \prime}$. This ring-like emission is similar to that found in NGC 1097. SJL95 also identify the existence of a jet-like structure originating from the nucleus and about $5^{\prime \prime}$ long in position angle (PA) $125^{\circ}$ (i.e., aligned with the minor axis of the galaxy). We observe a similar structure in our map. The detailed study of the spectral data (SJL95) indicate that both the jet and the nucleus have a steep spectral index. This jet-like feature appears to be aligned with the axis of the ionized gas. NGC 1365 has also been observed with the PTI at $13 \mathrm{~cm}\left(\sim 0.1^{\prime \prime}\right.$ resolution) by Roy et al. (1994) (hereafter R94) and S95 in which a 4 mJy component was detected. This object has been extensively studied in HI by Jörsäter \& van Moorsel (1995).

NGC 1320 (Mrk 607): This object is unresolved in our VLA observations. PTI observations place an upper limit of $S<4$ mJy on the compact flux density at $13 \mathrm{~cm}$ (R94).

NGC 1386: Ulvestad \& Wilson (1984b) observed this object at 20 and $6 \mathrm{~cm}$. In their observations, NGC 1386 is barely resolved with an extension to the southwest $\left(\mathrm{PA}=-125^{\circ}\right)$, and it is unresolved in our $3.5 \mathrm{~cm}$ map. Ulvestad \& Wilson (1984b) give a flux density of $13 \mathrm{mJy}$ and $23.0 \mathrm{mJy}$ at 6 and $20 \mathrm{~cm}$, respectively, and a spectral index of $\alpha_{20}^{6}=-0.47$. The spectral index between 6 and $3 \mathrm{~cm}$ is very flat, $\alpha_{6}^{3}=-0.05$. PTI observations find a flux density of $4 \mathrm{mJy}$ at $13 \mathrm{~cm}$ (R94, S95). 


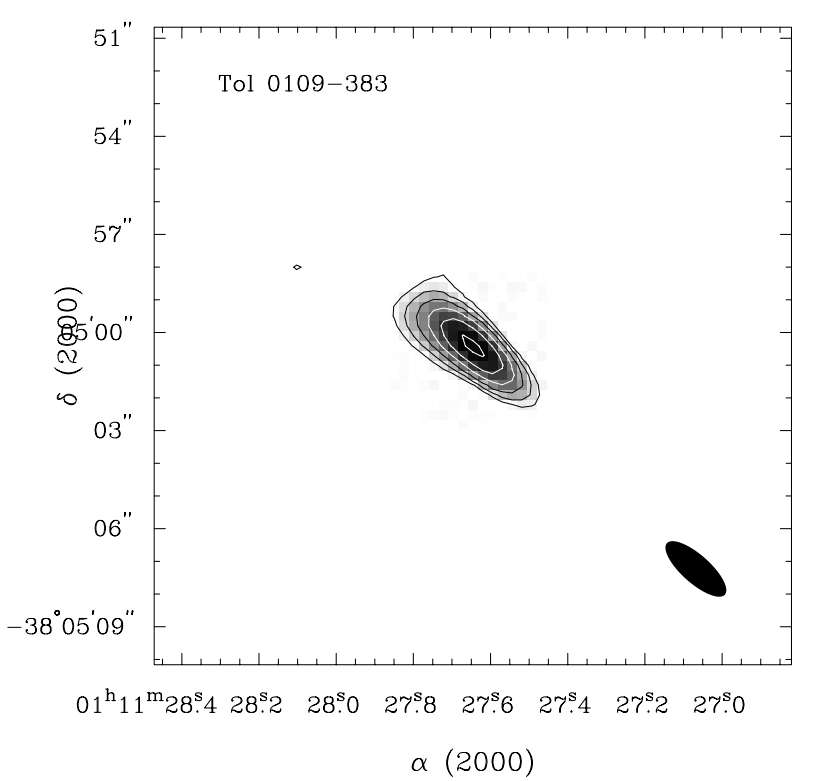

Fig. 5. TOL 0109-383. Contours: $-1.0,-0.6,0.6,1,1.7,3,5$, 9 mJy beam $^{-1}$

NGC 1566: A possible faint blob of radio emission is detected $3^{\prime \prime}$ north of central peak (Fig. 7). Observations with the PTI at $13 \mathrm{~cm}$ (R94 and S95) give a 5 mJy flux.

ESO 362-G18: This object is not resolved by our observations. PTI observations give an upper limit of $S<$ $4 \mathrm{mJy}$ on the flux density at $13 \mathrm{~cm}$ (R94). ESO $362-\mathrm{G} 18$ has been studied in [O III] $\lambda 5007$ and $\mathrm{H} \alpha$ by Mulchaey et al. (1996).

NGC 3281: This object is possibly barely resolved (along the north-south direction) in our data (Fig. 8), and was not resolved by Ulvestad \& Wilson (1989). Ulvestad \& Wilson (1989) find a flux of $61.2 \mathrm{mJy}$ at $20 \mathrm{~cm}$ and $26.7 \mathrm{mJy}$ at $6 \mathrm{~cm}$ which gives a steep spectral index of $\alpha_{20}^{6}=-0.65$. Our observations show that the spectral index remains steep out to $3 \mathrm{~cm},\left(\alpha_{6}^{3}=-0.83\right)$.

NGC 3393: This object was observed with both the VLA and ATCA. The two radio maps are very similar and show a double structure. Here we show only the VLA map (Fig. 2), which has a slightly better beam shape. From the two frequencies we derive a spectral index of $\alpha_{6}^{3}=-0.71$ for the NE side and -0.93 for the SW side. The NLR is elongated along a similar position angle as the radio $-\mathrm{PA}_{\mathrm{NLR}} \approx \mathrm{PA}_{\text {radio }}=45^{\circ}-$ with a close correspondence to the radio morphology. This is very clear in the HST image (Pogge 1997) which shows a spectacular S-shape morphology. A core of $16 \mathrm{mJy}$ was detected with the PTI at $13 \mathrm{~cm}$ (R94).

NGC 3660: This object is unresolved in our observations. The source was previously detected by the Effelsberg $100 \mathrm{~m}$ telescope (Kollatschny et al. 1983). The total flux

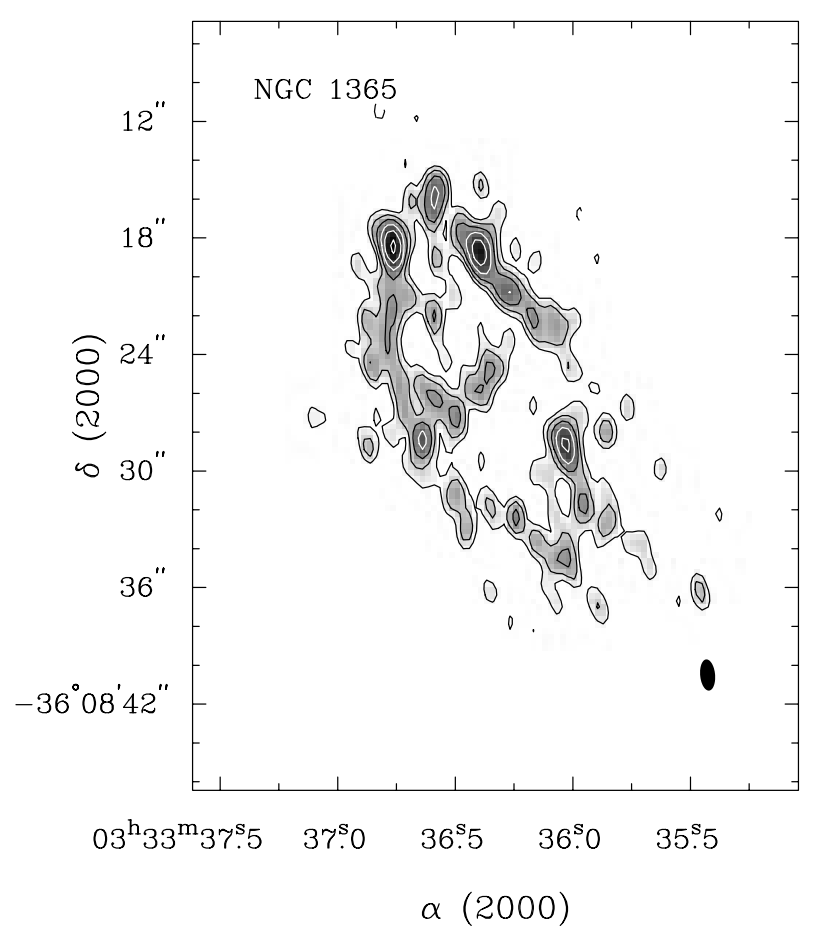

Fig. 6. NGC 1365. Contours: $-0.6,-0.4,0.4,0.6,0.9,1.5,2.3$, $3.6 \mathrm{mJy}$ beam $^{-1}$

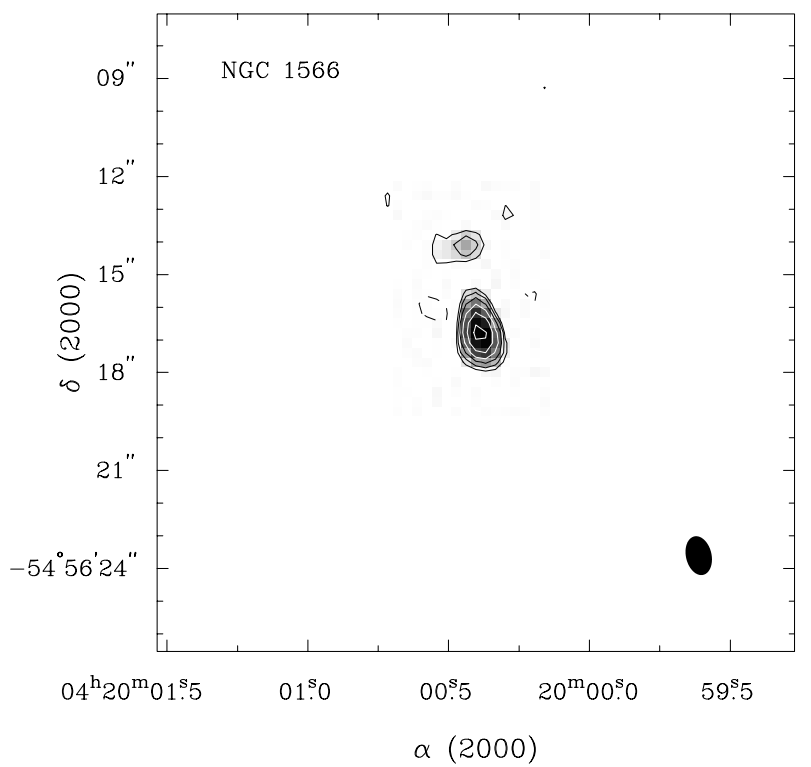

Fig. 7. NGC 1566. Contours: $-0.9,-0.6,0.6,0.9,1.4,2.2,3.4$, $5.3 \mathrm{mJy}$ beam $^{-1}$

measured in our observation $(0.5 \mathrm{mJy})$ is much smaller than that reported by Kollatschny et al. (11 mJy). The reason for this discrepancy is likely to be the presence of other two bright unrelated sources in the field. These sources could not be separated from NGC 3660 by the $100 \mathrm{~m}$ telescope but they are in our VLA data.

NGC 3783: The object is unresolved in our $8 \mathrm{GHz}$ observations and was also reported unresolved by Ulvestad 


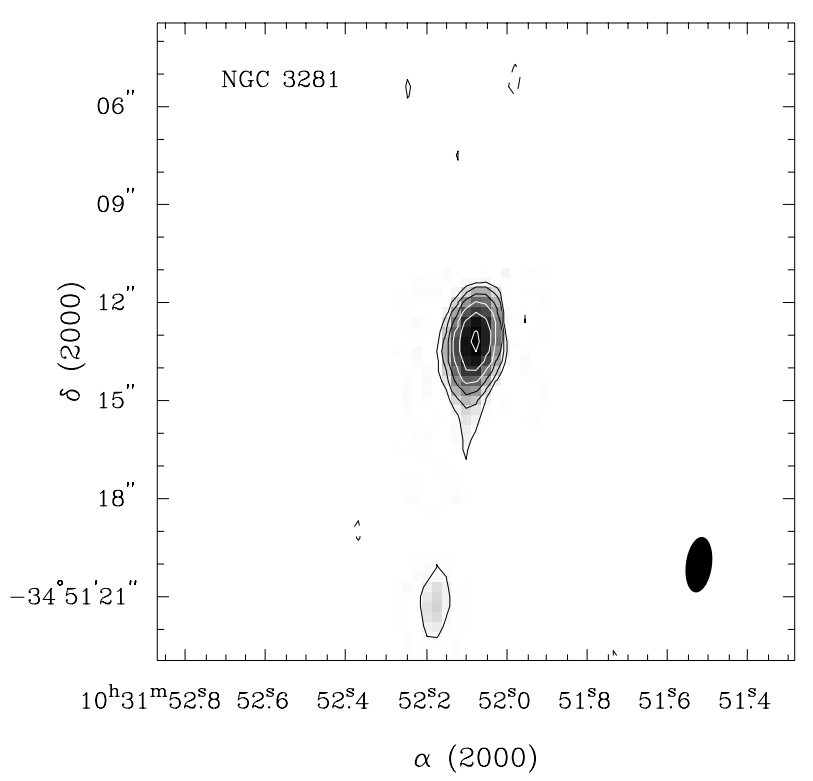

Fig. 8. NGC 3281. Contours: $-0.9,-0.5,0.5,0.9,1.9,3.7,7.2$, $14.2 \mathrm{mJy}$ beam $^{-1}$

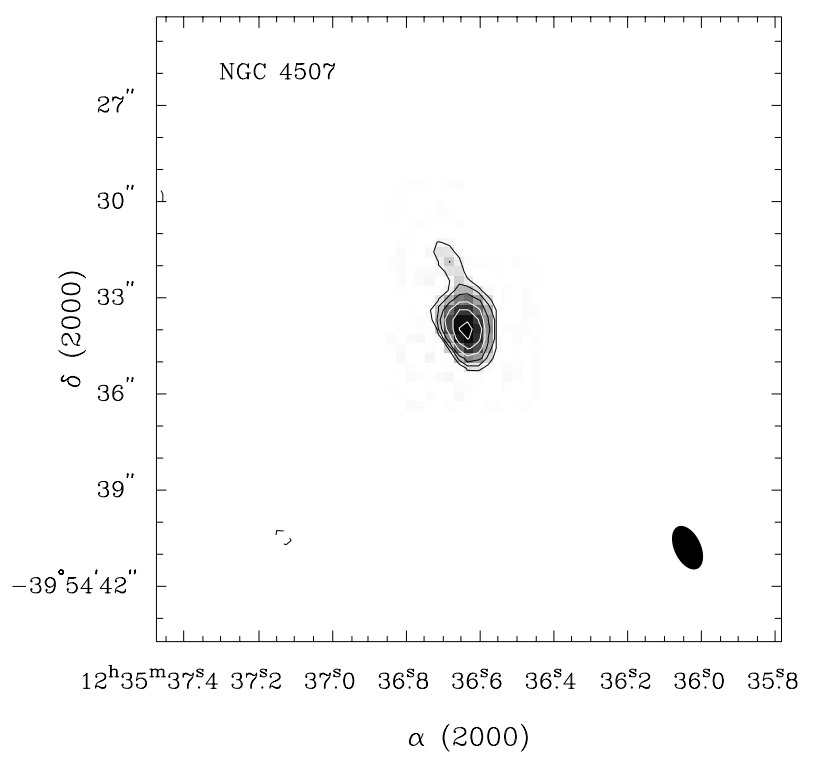

Fig. 9. NGC 4507. Contours: $-0.7,-0.5,0.5,0.71,1.4,2$, 3 mJy beam ${ }^{-1}$

\& Wilson (1984b) who give a flux density of $13 \mathrm{mJy}$ at $6 \mathrm{~cm}$. A spectral index of $\alpha_{6}^{3}=-0.55$ is derived from Ulvestad \& Wilson (1984b) and our flux measurements. An upper limit $(S<5 \mathrm{mJy})$ was obtained from the PTI observations at $13 \mathrm{~cm}$ (R94).

NGC 4507: In our $3 \mathrm{~cm}$ data, this object is slightly extended along $\mathrm{PA} \sim 10^{\circ}$ (Fig. 9). This face-on galaxy shows an high excitation NLR with a fairly symmetric edge-brightened bicone shape.

TOL 1238-364 (IC 3639): In our $3 \mathrm{~cm}$ map the source is unresolved. TOL $1238-364$ object was observed at 20 and $6 \mathrm{~cm}$ by Ulvestad \& Wilson (1989). At $20 \mathrm{~cm}$ the source

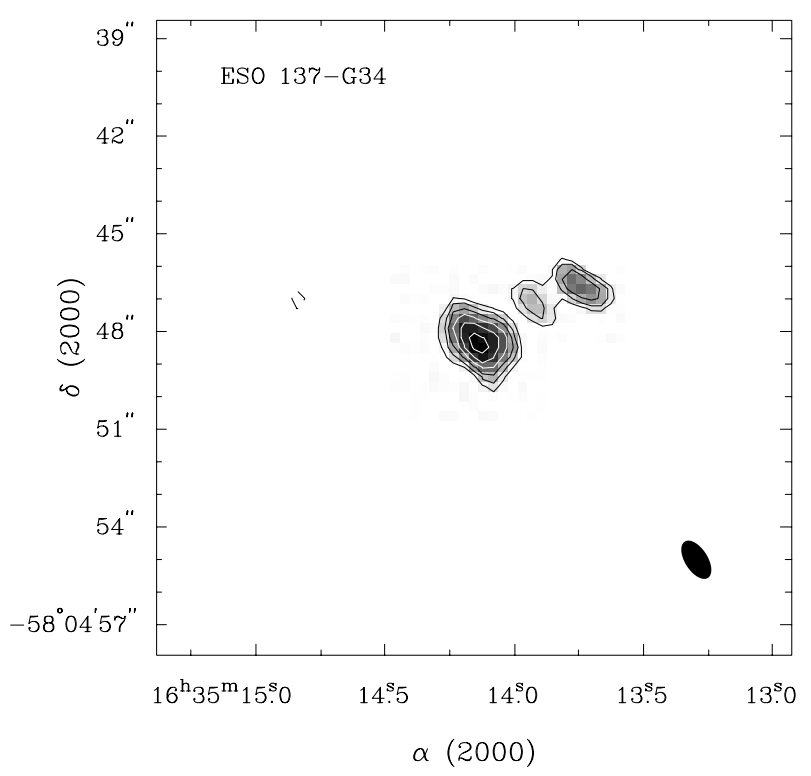

Fig. 10. ESO 137-G34 Contours: -0.5, -0.4, 0.4, 0.5, 0.7, 1, 1.3, 1.8 mJy beam $^{-1}$

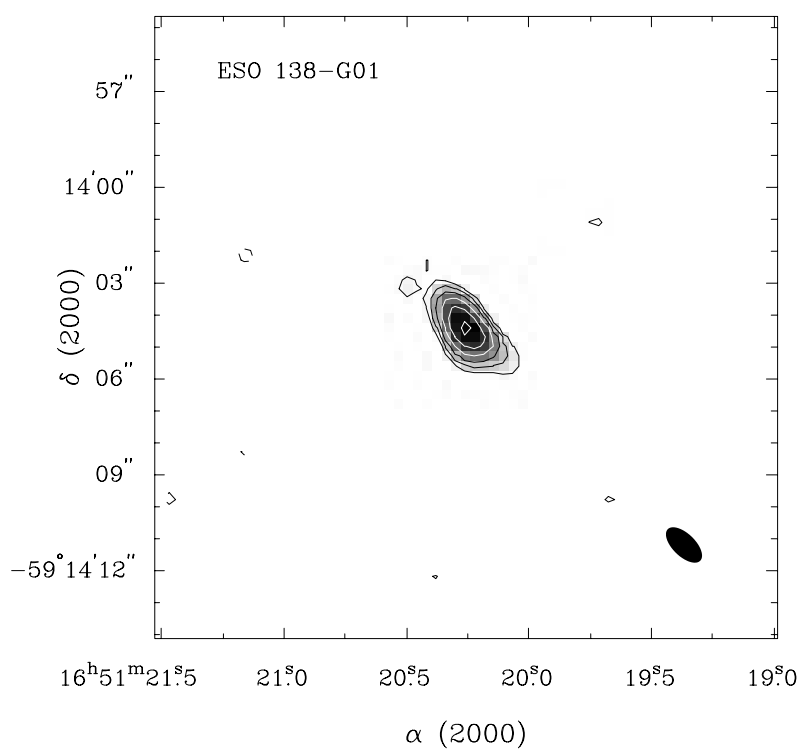

Fig. 11. ESO138-G01 Contours: -0.7, -0.4, 0.4, 0.7, 1.1, 1.6, 2.5, 3.9 mJy beam $^{-1}$

appears to have a diffuse emission around a relatively strong core. At $6 \mathrm{~cm}$ only the core of the source was detected. Ulvestad \& Wilson (1989) find a $6 \mathrm{~cm}$ flux of $13.6 \mathrm{mJy}$ which combined with our data gives a spectral index of $\alpha_{6}^{3}=-0.53$. A core of $13 \mathrm{mJy}$ was detected with the PTI at $13 \mathrm{~cm}$ (R94).

NGC 4968: This object is unresolved in our observations. A core of 10 mJy has been detected with the PTI at $13 \mathrm{~cm}$ (R94).

MCG-6-30-15: This is an unresolved and barely detected object. Ulvestad \& Wilson (1984b) reported a flux density of $1 \mathrm{mJy}$ at $6 \mathrm{~cm}$ and 1.7 at $20 \mathrm{~cm}$, giving 


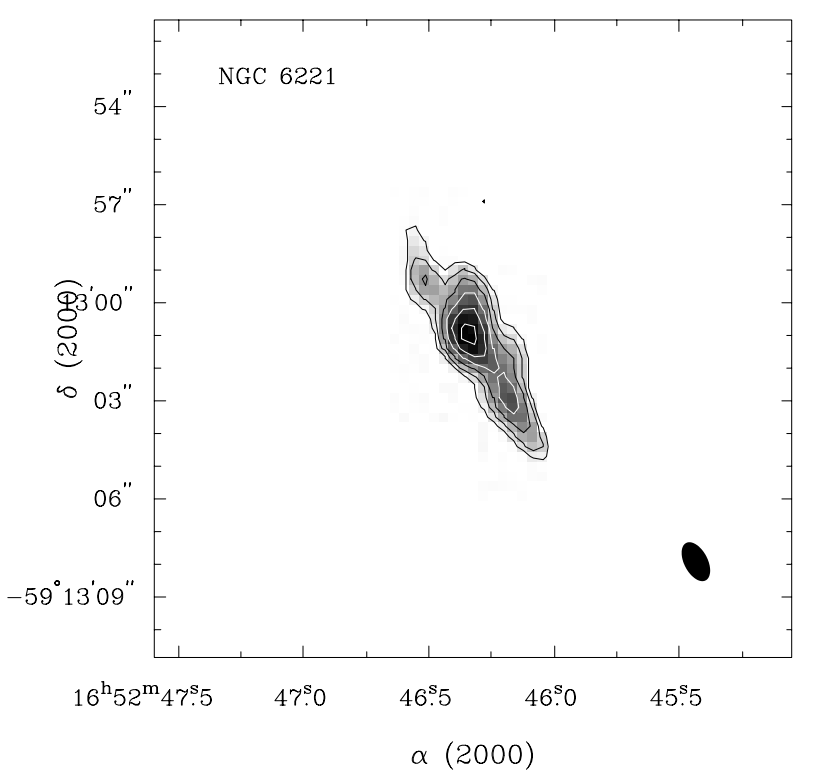

Fig. 12. NGC 6221 Contours: $-0.6,-0.4,0.4,0.6,0.9,1.3,1.8$, $2.7 \mathrm{mJy} \mathrm{beam}^{-1}$

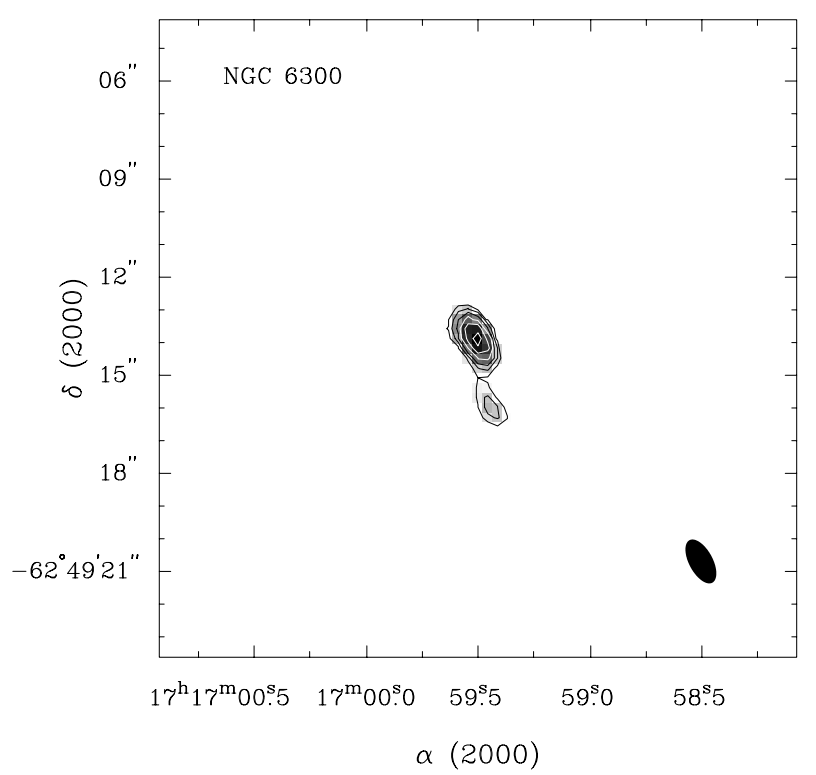

Fig. 13. NGC 6300 Contours: $-0.7,-0.5,0.5,0.7,0.9,1.1,1.4$, $1.8 \mathrm{mJy} \mathrm{beam}^{-1}$

a spectral index of $\alpha_{6}^{20}=-0.44$. The flux density from our observations give an inverted spectral index at high frequencies $\alpha_{6}^{3}=0.24$. The upper limit to the flux with the PTI is $S<4 \mathrm{mJy}$ at $13 \mathrm{~cm}$ (R94).

ESO 137-G34: This is a low-surface brightness spiral classified as Seyfert 2. The radio morphology (Fig. 10) consists of three knots aligned along $\mathrm{PA}_{\text {radio }} \sim-40^{\circ}$. The ionized gas has an $S$-shape morphology within a larger scale bi-cone. The radio emission is coincident with the inner (linear) part of the line-emitting gas.

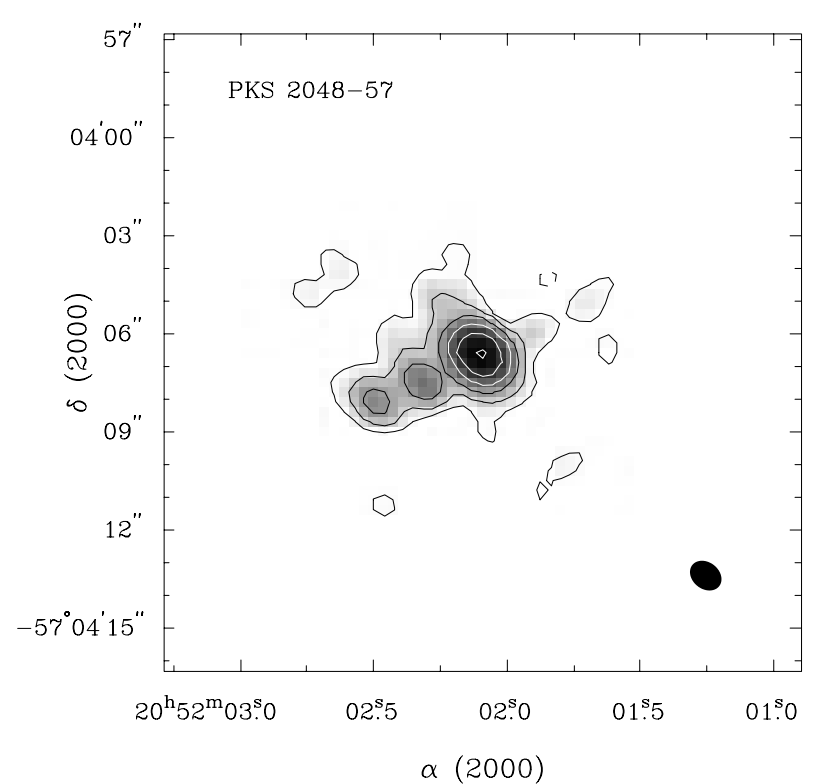

Fig. 14. IC 5063 Contours: $-1.8,-0.6,0.6,1.8,5.5,16.3,48.4$, 144.1 mJy beam $^{-1}$

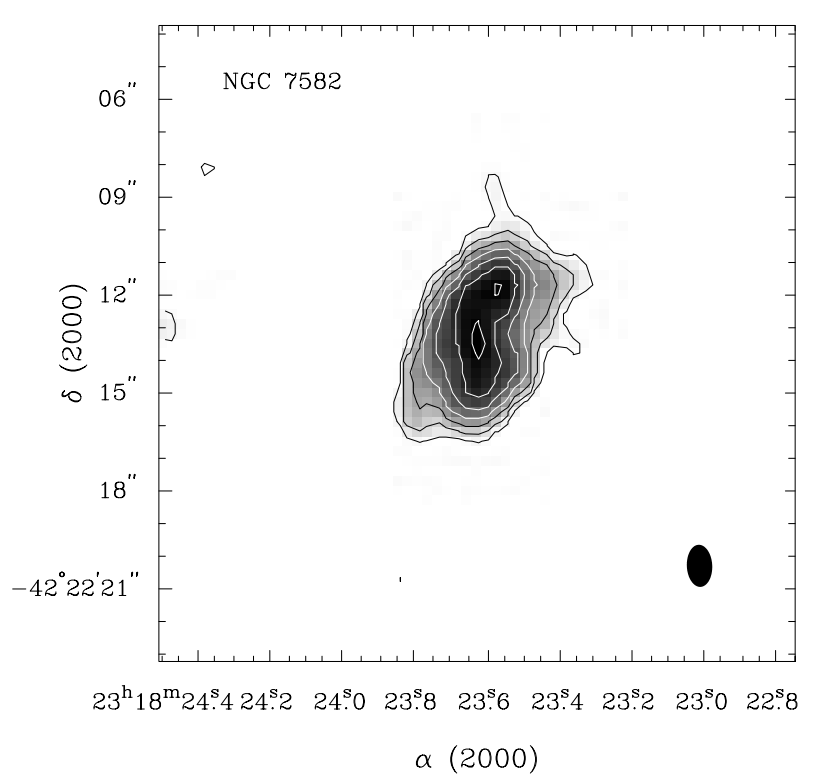

Fig. 15. NGC 7582 Contours: $-0.6,-0.4 \mathrm{~m} \mathrm{0.4,} \mathrm{0.6,} \mathrm{0.9,} \mathrm{1.5,}$ $2.3,3.6,5.6$ mJy beam $^{-1}$

ESO 138-G01: This is a Seyfert 2 nucleus residing in early type host galaxy. The radio emission (Fig. 11) is possibly elongated in $\mathrm{PA}_{\text {radio }} \sim 35^{\circ}$. The NLR has a jet-like morphology elongated $\mathrm{PA}_{\mathrm{NLR}} \sim-40^{\circ}$ The radio emission is also misaligned by $\sim 50^{\circ}$ with respect to the major axis of the galaxy.

NGC 6221: We resolve a radio triple (see Fig. 12) aligned along $\mathrm{PA}_{\text {radio }} \sim 40^{\circ}$. The upper limit to the core flux with the PTI at $13 \mathrm{~cm}$ is $S<2$ mJy (S94). 
NGC 6300: The radio morphology (Fig. 13) of this object is slightly elongated.

IC 5063 (PKS 2048-57): This is an early-type galaxy hosting a Seyfert 2 (Colina et al. 1991). Its radio luminosity is nearly 100 times larger than typical values for nearby Seyferts. Our ATCA radio map (Fig. 14, see also Morganti et al. 1998) resolves a linear radio structure comprising three compact features aligned along $\mathrm{PA}_{\text {radio }}=305^{\circ}$. The spectral index is steep: $\alpha_{6}^{20}=-1.1$ (Danziger et al. 1981). The optical line-emitting gas traces an X-shape (Morganti et al. 1998), and the radio emission coincides with the inner part of the line emitting region. Recent HI observations with ATCA and VLBI reveal a well defined H I disk aligned with a morphologically similar system of dust lanes (Morganti et al. 1998; Oosterloo et al. 1998). PTI observations measure a flux of $140 \mathrm{mJy}$ at $13 \mathrm{~cm}$ and $10 \mathrm{mJy}$ at $3 \mathrm{~cm}$ Slee et al. (1994).

NGC 7172: This object has a linear radio structure (Figs. 3 and 4) which is elongated roughly east-west $\left(\mathrm{PA}_{\text {radio }}=90^{\circ}\right)$. A $13 \mathrm{~cm}$ core flux of $3 \mathrm{mJy}$ has been detected with the PTI (R94).

IC 5201: This source is undetected in our observations. An upper limit $S<5$ mJy has been obtained from PTI observations at $13 \mathrm{~cm}$ (S94).

NGC 7496: This source is unresolved by our observations. A core flux of 7 mJy has been detected with the PTI at $13 \mathrm{~cm}$ (R94).

NGC 7582: Ulvestad \& Wilson (1984b) detected a weak core surrounded by an extended emission. They measure $69 \mathrm{mJy}$ at $6 \mathrm{~cm}$ and $166 \mathrm{mJy}$ at $20 \mathrm{~cm}$, giving a spectral index of -0.73 . We find (Fig. 15) that the spectral index remains steep out to $3 \mathrm{~cm}$ with $\alpha_{6}^{3}=-0.72$. For comparison, the NLR is a good example of an edgebrightened, wide angle bi-cone with a cone axis projected along $\mathrm{PA}_{\mathrm{e} .1 .}=55^{\circ}$. In addition, the host galaxy has a prominent bar along $\mathrm{PA}_{\mathrm{bar}}=155^{\circ}$. An upper limit to the flux density of $S<6 \mathrm{mJy}$ has been set by the PTI observations (S95, R94).

NGC 7590: This object is not detected in our data; the upper limit to the flux density from PTI is $S<3 \mathrm{mJy}$ at $13 \mathrm{~cm}$ (R94).

\section{Discussion}

The new radio observations presented in this paper cover more than $50 \%$ of our southern $\left(\delta<0^{\circ}\right)$ volume limited $\left(c z<3600 \mathrm{~km} \mathrm{~s}^{-1}\right)$ sample of Seyfert galaxies; adding data from the literature increases the coverage to almost
$85 \%$. There remain 9 sources in the southern sample for which radio information at arcsec resolution is not available yet.

Our survey largely overlaps with the samples studied by Ulvestad \& Wilson (1984b) and Ulvestad \& Wilson (1989). We do not, therefore, expect results completely independent from theirs. We note, however, that the sample of Ulvestad \& Wilson contained all Seyferts with $c z<3600 \mathrm{~km} \mathrm{~s}^{-1}$ known at the time of their observations $(\sim 15$ years ago), and was declination-limited by the VLA horizon restrictions. Our sample, on the other hand, covers all Seyferts with reliable classification to date at southern declinations $\left(\delta<0^{\circ}\right)$ and increases the distancelimited sample of Ulvestad \& Wilson (1989) by $22 \%$.

However, in order to further improve the statistics, we have collected additional sources from the literature, consisting of known Seyferts with $c z<4600 \mathrm{~km} \mathrm{~s}^{-1}$, derived mainly from Ulvestad \& Wilson (1989) and references therein. The selected sources are listed in Table 4. For this compilation, we selected only radio data of similar observing frequency and resolution to our observations $\left(\sim 0.3-1^{\prime \prime}\right)$. Most of the collected data were obtained at $6 \mathrm{~cm}$. The combined sample includes 71 objects of which 17 are Seyfert 1's and 54 Seyfert 2's.

Here we concentrate on the discussion of the radio characteristics of the studied sample. A discussion on the comparison between the radio and optical properties will be done in a forthcoming paper.

\subsection{Radio spectra}

We derived the spectral indices for most of our sources by combining our data with previous observations (see Sect. 3.1). The exception is NGC 3393, for which we use the 3 and $6 \mathrm{~cm}$ data presented here. In three cases (NGC 1097, NGC 1365 and IC 5063), we are unable to estimate the spectral index owing to complex source structure and poor matches in $(u, v)$ coverage. For NGC 1097 and NGC 1365 detailed studies of the spectral indices are already available (Hummel et al. 1987; Sandqvist et al. 1995, respectively).

In three cases (NGC 7582, NGC 3281 and Tol 0109-383) we find that the spectral index at high frequencies $\left(\alpha_{\text {high }}\right.$, between 3 and $\left.6 \mathrm{~cm}\right)$ is similar to the value at lower frequencies $\left(\alpha_{\text {low }}\right)$; that is, the spectra are steep in the first two cases, and flat for Tol 0109-383. In two cases (MGC-6-30-15 and NGC 1386) the spectra appear to flatten with increasing frequency $\left(\alpha_{\text {high }}>\alpha_{\text {low }}\right)$, although the radio source in MGC-6-30-15 is very weak, and the derived spectral index is uncertain. New spectral indices have been derived for NGC 3393, NGC 3783 and Tol 1238-364: in all these cases the spectral index is steep $(\alpha<-0.5)$. For comparison, most of the Seyfert galaxies have steep radio spectra, but flat-spectrum cores are found in a few Seyferts (Wilson 1991). 
Table 4. Radio parameters from the literature

\begin{tabular}{|c|c|c|c|c|c|c|c|}
\hline Object & $\begin{array}{r}c z \\
\mathrm{~km} \mathrm{~s}^{-1} \\
\end{array}$ & $\begin{array}{r}\text { Sey } \\
\text { Type }\end{array}$ & $\begin{array}{r}S_{6 \mathrm{~cm}} \\
\mathrm{mJy}\end{array}$ & $\begin{array}{c}\log P_{6 \mathrm{~cm}} \\
\mathrm{~W} \mathrm{~Hz}\end{array}$ & $\begin{array}{r}\text { Radio } \\
\text { Morph. }\end{array}$ & $\begin{array}{l}\text { Size } \\
\text { kpc }\end{array}$ & Refs. \\
\hline NGC 2639 & 3336 & 1 & 54.5 & 22.11 & $\mathrm{~L}$ & 0.41 & 4 \\
\hline NGC 3227 & 1157 & 1 & 34.0 & 20.99 & S & 0.30 & 3 \\
\hline NGC 3516 & 2649 & 1 & 4.3 & 20.81 & $\mathrm{U}$ & $<0.05$ & 3 \\
\hline NGC 3786 & 2678 & 1 & 3.4 & 20.72 & $\mathrm{~S}$ & 0.28 & 3 \\
\hline NGC 4051 & 725 & 1 & 6.0 & 19.83 & L-D? & 0.57 & 3 \\
\hline NGC 4151 & 995 & 1 & 125.0 & 21.42 & $\mathrm{~L}$ & 0.34 & 3,5 \\
\hline NGC 4235 & 2410 & 1 & 5.3 & 20.82 & $\mathrm{U}$ & $<0.05$ & 3 \\
\hline NGC 4253 & 3876 & 1 & 19.5 & 21.80 & $\mathrm{U}$ & $<0.35$ & 4 \\
\hline NGC 4593 & 2698 & 1 & 1.6 & 20.40 & $\mathrm{U}$ & $<0.04$ & 3 \\
\hline NGC 5033 & 875 & 1 & 3.3 & 19.73 & $\mathrm{U}$ & $<0.02$ & 4 \\
\hline NGC 5273 & 1054 & 1 & 0.9 & 19.33 & S & 0.10 & 3 \\
\hline NGC 6814 & 1563 & 1 & 2.2 & 20.06 & $\mathrm{~S}$ & 0.03 & 3 \\
\hline 0714-2914 & 1630 & 2 & 28.2 & 21.20 & $\mathrm{~L}$ & 0.55 & 4 \\
\hline $0942+0950$ & 3897 & 2 & $<0.4$ & $<20.11$ & $\mathrm{U}$ & $<0.10$ & 4 \\
\hline Mrk 3 & 4050 & 2 & 361.0 & 23.10 & $\mathrm{~L}$ & 0.58 & 2,6 \\
\hline Mrk 270 & 2700 & 2 & 5.7 & 20.95 & $\mathrm{~L}$ & 0.61 & 2 \\
\hline Mrk 348 & 4540 & 2 & 480.0 & 23.32 & $\mathrm{~L}$ & 0.06 & 2,7 \\
\hline Mrk 1066 & 3605 & 2 & 35.5 & 21.99 & $\mathrm{~L}$ & 0.70 & 4 \\
\hline NGC 0591 & 4547 & 2 & 7.9 & 21.54 & $\mathrm{~L}$ & 0.18 & 4 \\
\hline NGC 0788 & 4078 & 2 & 1.2 & 20.63 & $\mathrm{U}$ & $<0.08$ & 4 \\
\hline NGC 1068 & 1136 & 2 & 1090.0 & 22.48 & $\mathrm{~L}$ & 1.43 & 1,8 \\
\hline NGC 1358 & 4013 & 2 & 1.2 & 20.62 & S & 0.10 & 4 \\
\hline NGC 1667 & 4547 & 2 & 1.0 & 20.64 & S & 0.12 & 4 \\
\hline NGC 1685 & 4527 & 2 & 5.3 & 21.37 & S & 0.12 & 4 \\
\hline NGC 2273 & 1840 & 2 & 19.0 & 21.14 & $\mathrm{~L}$ & 0.24 & 3 \\
\hline NGC 3081 & 2385 & 2 & 0.9 & 20.04 & $\mathrm{U}$ & $<0.06$ & 3 \\
\hline NGC 3982 & 1109 & 2 & 2.2 & 19.76 & $\mathrm{U}$ & $<0.03$ & 4 \\
\hline NGC 4117 & 871 & 2 & $<0.6$ & $<18.99$ & S & $<0.08$ & 4 \\
\hline NGC 4388 & 2524 & 2 & 76.0 & 22.02 & $\mathrm{~L}$ & 3.92 & 3 \\
\hline NGC 4941 & 1108 & 2 & 4.3 & 20.05 & S & 0.10 & 3 \\
\hline NGC 5135 & 4112 & 2 & 58.8 & 22.33 & A & 1.97 & 4 \\
\hline NGC 5347 & 2335 & 2 & 2.2 & 20.41 & $\mathrm{U}$ & $<0.06$ & 4 \\
\hline NGC 5695 & 4225 & 2 & $<0.5$ & $<20.28$ & $\mathrm{U}$ & $<0.38$ & 4 \\
\hline NGC 5728 & 2788 & 2 & 4.6 & 20.88 & $\mathrm{~L} ?$ & 2.34 & 3,9 \\
\hline NGC 5929 & 2561 & 2 & 24.7 & 21.54 & $\mathrm{~L}$ & 0.18 & 3,10 \\
\hline NGC 6890 & 2419 & 2 & 4.2 & 20.72 & $\mathrm{U}$ & $<0.06$ & 4 \\
\hline NGC 7450 & 3191 & 2 & 1.7 & 20.57 & $\mathrm{~L}$ ? & 0.62 & 3 \\
\hline NGC 7672 & 4117 & 2 & 1.0 & 20.56 & $\mathrm{~L}$ & 1.86 & 4 \\
\hline Tol 0074 & 3285 & 2 & 13.6 & 21.50 & $\mathrm{D}$ & $<0.09$ & 4 \\
\hline MCG-5-23-16 & 2482 & 2 & 6.0 & 20.90 & S & 0.08 & 3 \\
\hline NGC 2110 & 2284 & 2 & 175.0 & 22.29 & $\mathrm{~L}$ & 0.87 & 3 \\
\hline NGC 2992 & 2311 & 2 & 77.0 & 21.94 & L-D? & 1.36 & 3,11 \\
\hline NGC 5506 & 1853 & 2 & 160.0 & 22.07 & S & 0.65 & 3 \\
\hline
\end{tabular}

References: (1) Wilson \& Ulvestad (1983); (2) Ulvestad \& Wilson (1984a); (3) Ulvestad \& Wilson (1984b); (4) Ulvestad \& Wilson (1989); (5) Pedlar et al. (1993); (6) Kukula et al. (1993); (7) Neff \& de Bruyn (1983); (8) Gallimore et al. (1996); (9) Shommer et al. (1988); (10) Su et al. (1996); (11) Wehrle \& Morris (1988).

Accepting uncertainties in our spectral index measurements due to mismatched $(u, v)$ coverage in the 3 and $6 \mathrm{~cm}$ maps, we measure a median spectral index steep $(\alpha \sim-0.67)$ for our Seyfert nuclei. This result is in agreement, with the spectral indices of the inner 0 l' $^{\prime} 1$ nuclear regions of spirals (and Seyferts) as measured by Sadler et al. (1995) using PTI observations. Their investigation of the compact radio cores in spiral and elliptical galaxies found a median spectral index for spirals (and Seyferts) of $\alpha=-1.0$, and that spirals usually have steeper core spectra than do elliptical galaxies (median spectral in$\operatorname{dex} \alpha=+0.27$ ). The flat (or inverted) spectral index of the cores is a typical characteristic of elliptical galaxies found on all scales in which the nuclear regions have been observed (from arcsec and sub-arcsec scale, see e.g. Morganti et al. 1997 and Slee et al. 1994 to VLBI scale). 
Thus, our study confirms the result that the spectral indices of Seyferts are much steeper than in the cores of ellipticals, and that the spectral index remains close to the same value over $0^{\prime \prime} 1$ to $1^{\prime \prime}$ scales.

In connection with this difference in spectral index, it is worth remembering that the nuclear regions of Seyfert galaxies appear to have a more complicated situation than in radio galaxies: following the detailed studies of few well known objects (e.g. NGC 1068, Gallimore et al. 1996; Roy et al. 1998; NGC 4151, Ulvestad et al. 1998) free-free absorption appears to be relevant in Seyfert to dim the "real" radio core. Thus the nuclear emission (and its spectral index) can be dominated not by the core itself but by bright blobs. This would be in agreement with the finding (Sadler et al. 1995) that in ellipticals most of the radio emission in the central kpc comes from the parsec-scale core, while in Seyferts this is only a small fraction $(10-25 \%)$.

\subsection{Radio structure}

Table 3 summarizes the classification of the radio morphology for the newly observed objects, following the scheme of Ulvestad \& Wilson (1984b). Table 4 provides source classifications for data taken from the literature. Including Seyferts from the literature, we find $38 \%$ of the sample are unresolved sources, $23 \%$ have slightly resolved structures and $34 \%$ have linear structure at arcsecond resolution; the remaining $5 \%$ have diffuse, amorphous, or ringed structures.

We can compare these numbers with the results from Ulvestad \& Wilson (1989) and references therein (see also the summary in Wilson 1991). Compared to Wilson (1991), we measure a higher fraction of unresolved sources and a lower fraction of galaxies with diffuse radio emission. This result may be due to an observational bias; our observations are not as sensitive to diffuse, extended, steep spectrum emission as are the $20 \mathrm{~cm}$ VLA observations of, e.g., Ulvestad \& Wilson (1989). Otherwise, we measure a similar detection fraction of linear or slightly resolved structures.

Linear radio sources in Seyfert galaxies generally trace radio outflows, but it is worth checking that linear radio emission is not associated with star formation in an edge-on galaxy. Among the linear radio sources detected in this survey, only the host galaxy NGC 7172 is near edgeon. The radio structure aligns with the plane of the host galaxy, casting doubt on a jet origin; rather, it appears that the linear radio source of NGC 7172 may be associated plausibly with a nuclear starburst. Otherwise, the remaining linear radio sources are more likely associated with an AGN-driven outflow.

To compare the distributions of radio power, we adjusted all of the radio luminosities to their $6 \mathrm{~cm}$ values. For sources not observed at $6 \mathrm{~cm}$, we adjusted the luminosities using measured spectral indices where possible.
Table 5. Log-linear fit to Radio Power vs. Size

\begin{tabular}{lrrrr}
\hline Method & Intercept & Err & Slope & Err \\
\hline Buckley-James & -11.36 & $\ldots$ & 0.51 & 0.09 \\
E-M & -12.31 & 2.25 & 0.55 & 0.11 \\
Schmitt & -12.72 & 2.20 & 0.56 & 0.10 \\
\hline
\end{tabular}

For those sources having neither $6 \mathrm{~cm}$ measurements or measured spectral indices, we assumed a spectral index of $\alpha_{6}^{3}=-0.5$. Many of the low power sources are unresolved, resulting in an upper limit to the source size. Since we are interested in the compact radio emission from jets rather than extended, low surface brightness emission (which may come from star-forming regions and starburstdriven superwinds), we also assumed that the three undetected sources were unresolved (size upper limit) and radio weak (flux upper limit). To account appropriately for these limits, we employed survival analysis techniques from the ASURV package (Feigelson \& Nelson 1985; Isobe et al. 1986) as it is implemented in IRAF.

We confirm a correlation between radio power and size (Fig. 16), originally discussed by Ulvestad \& Wilson (1984b) and Giuricin et al. (1990). Both the power-size and flux-angular size correlations are significant at better than $1 \%$ (probability of no correlation) according to the traditional survival analysis tests (Kendall's $\tau$, Spearman's $\rho$, and the Cox Proportional History model). A summary of log-linear models for the power-size correlation is provided in Table 5. Comparing with the results of Ulvestad \& Wilson (1984b), we find a steeper slope in the correlation owing to a proper treatment of limits at low radio-powers. Mrk 348 is an outlier, falling at relatively small size for its radio power. The present analysis ignores the $\sim 5$ kiloparsec radio lobes in this source, which may arise from either a starburst-driven superwind, or old nuclear-driven ejecta. Accounting for the extended radio lobes places Mrk 348 closer to the best-fit line, except that Mrk 348 now falls somewhat oversized for its luminosity according to the correlation.

\subsection{A Comparison of Seyfert $1 s$ and $2 s$}

Previous radio studies of volume-limited samples of Seyfert galaxies find: a) marginal or no statistically significant difference between the distributions of radio luminosities of Seyfert 1s and 2s; b) Seyfert 2's galaxies tend to have a larger radio sources than Seyfert 1s, although at $<90 \%$ significance level; c) the two types of Seyferts have essentially the same distribution of spectral indices (Giuricin et al. 1990; Rush et al. 1996) although there seems to be some evidence that flat spectrum cores are more common in Seyfert 1s than in Seyfert 2s (Ulvestad \& Wilson 1989); and d) compact radio cores (on the sub-arcsec scale) are more common in Seyfert 2s than in Seyfert 1s (R94). 


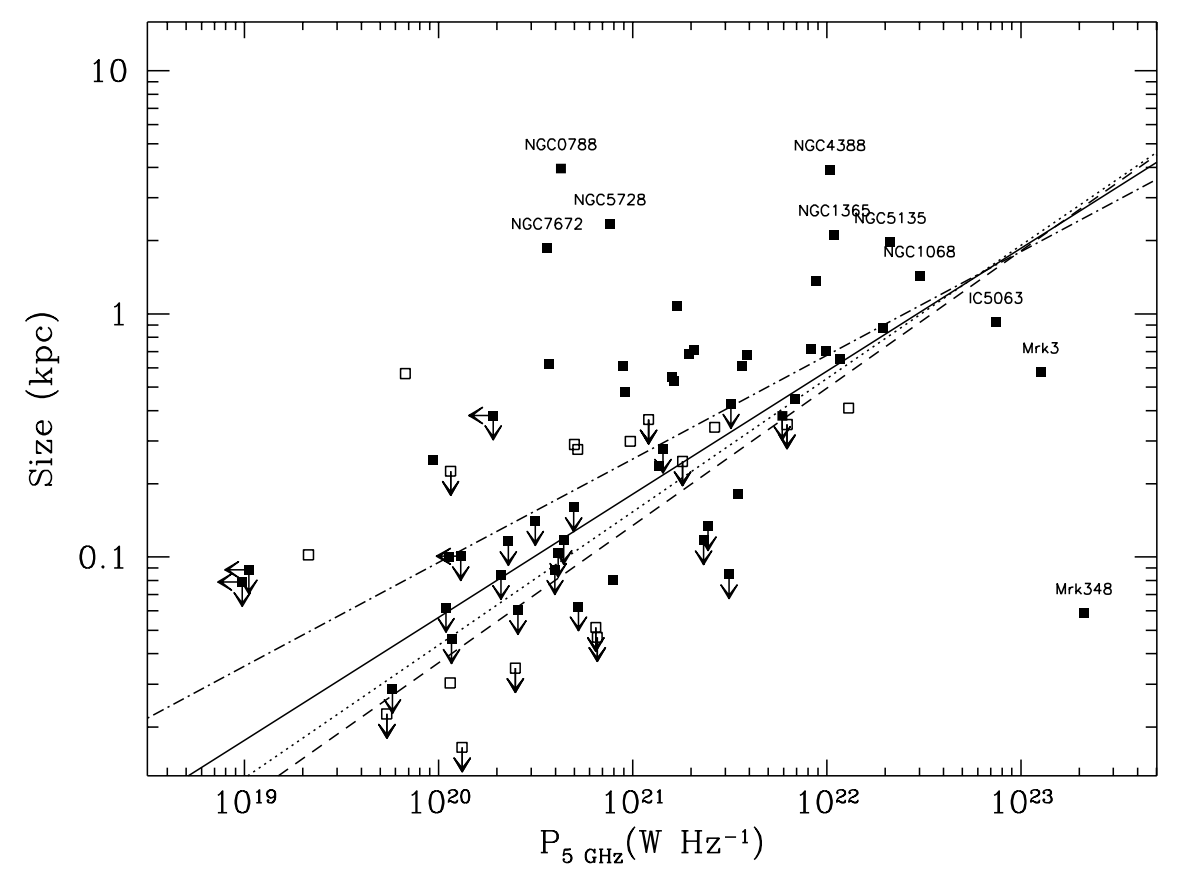

Fig. 16. Plot of the size of the radio emission versus the radio power (at $5 \mathrm{GHz}$ ): open squares represent Seyfert 1 and filled squares represent Seyfert 2

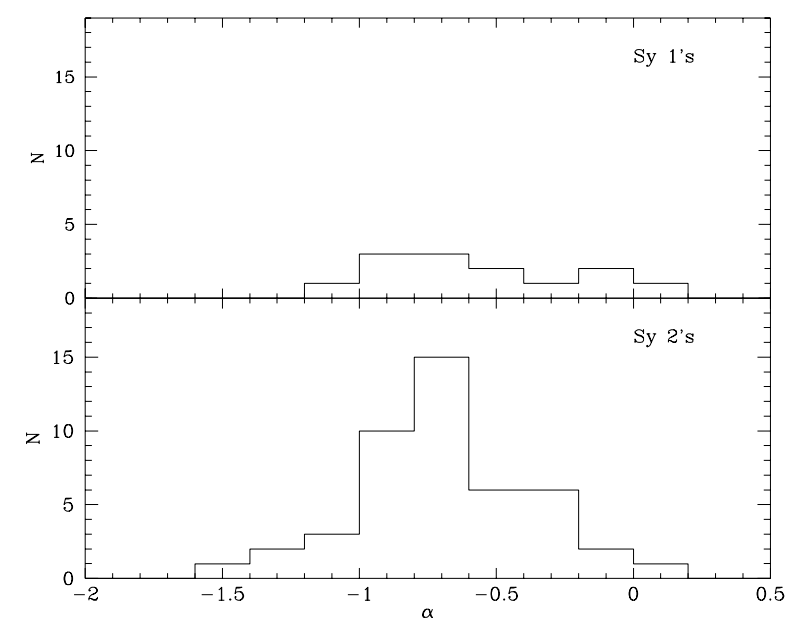

Fig. 17. Histograms of the spectral index distribution for Seyfert 1's and Seyfert 2's

Examining first the distribution of spectral index (see histograms in Fig. 17), we find a median value for the spectral index $\left(\alpha_{6}^{20}\right.$ or $\left.\alpha_{3}^{6}\right)$ of $\alpha=-0.44$ for Seyfert 1s and -0.72 for Seyfert $2 \mathrm{~s}$. However, the two distributions are not significantly different under a Kolmogorov-Smirnov test $(13 \%$ probability that the two distributions are not different). Unfortunately, we have too few Seyfert 1's with

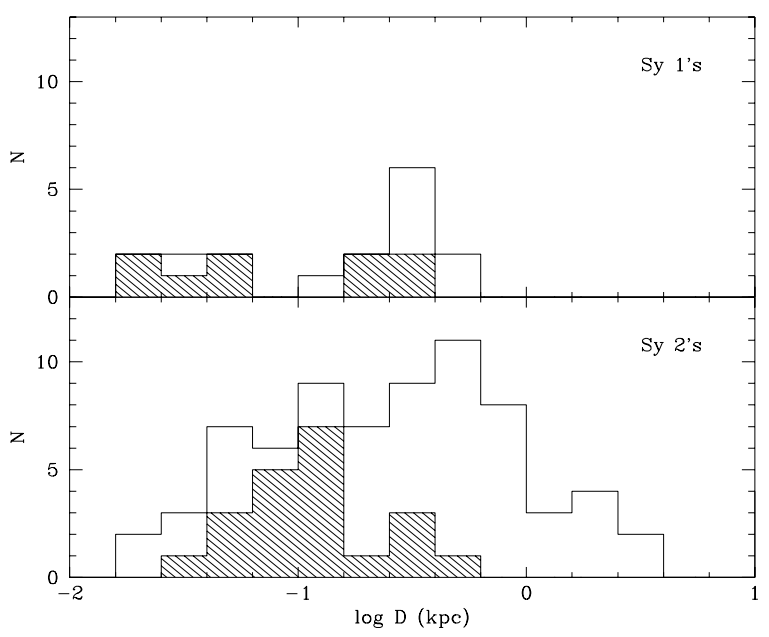

Fig. 18. Histograms of the radio size distribution for Seyfert 1's and Seyfert 2's. Shaded regions represent upper limits

measured spectral indices to compare the frequency of flatspectrum cores.

We also compared the distribution of radio sizes and powers (see histograms in Figs. 18 and 19 respectively) of Seyfert 1 and Seyfert 2 galaxies. To improve the fairness of the comparison, we only included sources out to a redshift of $c z=4000 \mathrm{~km} \mathrm{~s}^{-1}$, at which the redshift distributions of Seyferts 1 and 2 matched according to a K-S test. 


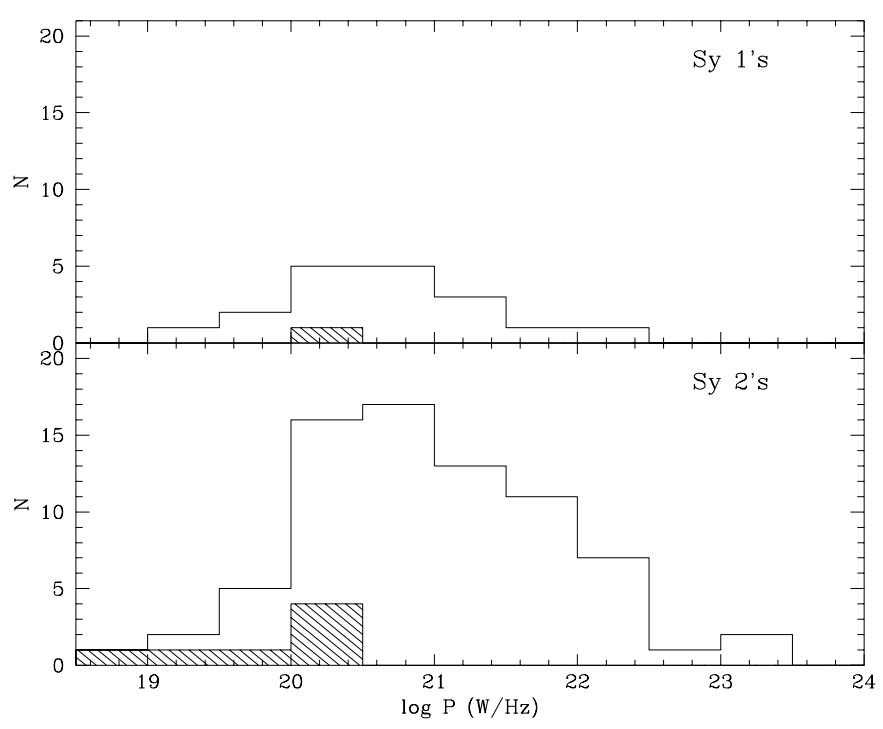

Fig. 19. Histograms of the radio power distribution for Seyfert 1 's and Seyfert 2's. Shaded regions represent upper limits

At marginal significance, we find that Seyfert 2 radio sources tend to be more luminous than Seyfert 1 radio sources; the probability that Seyferts 1 and 2 arise from the same parent distribution is $\sim 11 \%$ for most two-sample tests, 25\% for the logrank test. Ulvestad \& Wilson 1984a found a similar result for Markarian Seyferts, but they showed in a follow-up paper (Ulvestad \& Wilson 1989) that the significance is reduced owing to the paucity of low-luminosity Seyfert $2 \mathrm{~s}$ in the Markarian sample. Inspection of the power-size diagram (Fig. 12) suggests that any significance in the difference of radio powers owes to a handful of luminous Seyfert $2 \mathrm{~s}$, but most Seyferts 1 and 2 have comparable radio powers. Formally, we measure mean $\log$ radio powers of $\log P\left(\mathrm{~W} \mathrm{~Hz}^{-1}\right)=21.03 \pm$ 0.01 for Seyfert $2 \mathrm{~s}$, and $\log P\left(\mathrm{~W} \mathrm{~Hz}^{-1}\right)=20.66 \pm 0.18$ for Seyfert 1s.

In contrast, we measure a significant difference in radio sizes. All of the two-sample tests report a difference in parent populations to a significance of $\geq 95 \%$, with the exception of the logrank test, which is significant only to $87 \%$. Seyfert $2 \mathrm{~s}$ tend to be larger, with a mean size of $0.53 \pm 0.12 \mathrm{kpc}$, compared to Seyfert 1s, mean size $0.16 \pm 0.04 \mathrm{kpc}$. The errors on the mean sizes represent the dispersion in the intrinsic value. Given the statistical agreement in the distribution of radio powers, it is natural to interpret the size difference in terms of an orientation unifying scheme. The prediction of this model is the nuclear axes of Seyfert 1s are viewed more nearly pole-on, and so radio jets are foreshortened by projection.

\section{Conclusions}

We have presented VLA $6 \mathrm{~cm}$ and ATCA $3.5 \mathrm{~cm}$ radio data for 29 southern Seyfert galaxies selected from a volume limited sample $\left(c z<3600 \mathrm{~km} \mathrm{~s}^{-1}\right)$. The resolution of the observations is about $\sim 1^{\prime \prime}$, similar to that of the ground-based, optical narrow band images. The new radio observations presented in this paper cover more than $50 \%$ of our southern $\left(\delta<0^{\circ}\right)$ volume limited $\left(c z<3600 \mathrm{~km} \mathrm{~s}^{-1}\right)$ sample of Seyfert galaxies. When data available from the literature are added the coverage improves to greater than $80 \%$. Only 2 of the 22 newly observed sources are undetected in our radio observations. Including data from the literature, we find $38 \%$ of the distance-limited sample is unresolved at arcsecond resolution, and most of the resolved objects ( $34 \%$ of the sample) show, as expected, a linear structure.

Statistical comparisons are consistent with the picture that Seyferts 1 and 2 arise from the same parent population of AGN, but the radio jets of Seyfert 1 galaxies are smaller in projection owing to their orientation. This model is not contrary to the power-size correlation, but, conversely, projection effects add noise to the measured correlation. An additional prediction is that, binned in radio power, Seyfert 1s should be smaller than Seyfert 2s. Unfortunately, owing to the small number of sources per decade in radio power and the large number of limits at low radio power, we are unable to test this prediction with the current sample.

Acknowledgements. MA acknowledges the support of an Australian Post-Graduate Research Award (APRA). This research has made use of the NASA/IPAC Extragalactic Database (NED) which is operated by the Jet Propulsion Laboratory, Caltech, under contract with NASA.

\section{References}

Baars J.W.M., Genzel R., Pauliny-Toth I.I.K., Witzel A., 1977, A\&A 61,99

Capetti A., Axon D.J., Macchetto F., Sparks W.B., Boksenberg A., 1996, ApJ 469, 554

Colina L., Sparks W.B., Macchetto F., 1991, ApJ 370, 102

de Bruyn A.G., Wilson A.S., 1978, A\&A 64, 433

Danziger J.I., Goss W.M., Wellington K.J., 1981, MNRAS 196, 845

Edelson R.A., 1987, ApJ 313, 651

Feigelson E.D., Nelson P.I., 1985, ApJ 293, 192

Gallimore J.F., Baum S.A., O'Dea C.P., Pedlar A., 1996a, ApJ 458,136

Gallimore J.F., Baum S.A., O’Dea C.P., 1996b, ApJ 464, 198

Giuricin G., Mardirossian F., Mezzetti M., Bertotti G., 1990, ApJ 72, 551

Haniff C.A., Wilson A.S., Ward M.J., 1988, ApJ 334, 104

Hummel E., van der Hulst J.M., Keel W.C., 1987, A\&A 172, 32

Isobe T., Feigelson E.D., Nelson P.I., 1986, ApJ 306, 490

Jörsäter S., van Moorsel G.A., 1995, AJ 110, 2037

Kollatschny W., Fricke K.J., Biermann P., Huchtmeier W. Witzel A., 1983, A\&A 119, 80

Kukula M.J., Ghosh T., Pedlar A., Schilizzi R.T., Miley G.K., de Bruyn A.G., Saikia D.J., 1993, MNRAS 264, 893 
Meurs E.J.A., Wilson A.S., 1984, A\&A 136, 206

Morganti R., Oosterloo T.A., Tsvetanov Z., 1998, AJ 115, 915

Morganti R., Oosterloo T.A., Reynolds J., Tadhunter C.N., Migenes V., 1997, MNRAS 284, 541

Mulchaey J.S., Wilson A.S., Tsvetanov Z., 1996, ApJS 102, 309

Muxlow T.W.B., Pedlar A., Holloway A.J., Gallimore J.F., Antonucci R.R.J., 1996, MNRAS 278, 854

Neff S.G., de Bruyn A.G., 1983, A\&A 128, 318

Oosterloo T., Morganti R., Tzioumis A., Reynolds J., Tsvetanov Z., 1998, in IAU 164, Zensus A., Wrobel J.M., Taylor G.B. (eds.), p. 197

Pedlar A., Kukula M.J., Longley D.P.T., Muxlow T.W.B., Axon D.J., Baum S., O’Dea C., Unger S.W., 1993, MNRAS 263, 471

Pogge R.W., 1989, ApJ 345, 730

Pogge R.W., 1997, in "Emission Lines in Active Galaxies: New Methods and techniques", Peterson B.M., Cheng F.-Z., Wilson A.S. (eds.) ASP Conf. Ser. 113, 378

Reynolds J.E., 1996, in "Australia Telescope Compact Array User's Guide", Walsh W.M., McKay D.J. (eds.)

Roy A.L., Norris R.P., Kesteven M.J., Troup E.R., Reynolds J.E., 1994, ApJ 432, 496 (R94)

Roy A.L., Colbert E.J., Wilson A.S., Ulvestad J.S., 1998, ApJ 504,147

Rush B., Malkan M.A., Edelson R.A., 1996, ApJ 473, 130

Sault R.J., Teuben P.J., Wright M.C.H., 1995, in Astronomical Data Analysis Software and Systems. IV, Shaw R., Payne H.E., Hayes J.J.E. (eds.) ASP Conf. Ser. 77, 433

Sadler E.M., Slee O.B., Reynolds J.E., Roy A.L., 1995, MNRAS 276, 1373 (S95)
Sandqvist A., Jörsäter S., Lindblad P.O., 1995, A\&A 295, 585

Schommer R.A., Caldwell N., Wilson A.S., Baldwin J.A., Phillips M.M., Williams T.B., Turtle A.J., 1988, ApJ 324, 154

Slee O.B., Sadler E.M., Reynolds J.E., Ekers R.D., 1994, MNRAS 269, 928

Storchi-Bergmann T., Baldwin J.A., Wilson A.S., 1993, ApJ 410L, 11

Su B.M., Muxlow T.W.B., Pedlar A., Holloway A.J., Steffen W., Kukula M.J., Mutel R.L., 1996, MNRAS 279, 1111

Tsvetanov Z., Morganti R., Fosbury R.A.E., Allen M.G., Gallimore J., 1998, in "Looking Deep in the Southern Sky", ESO/Australia workshop, Morganti \& Couch (eds.). Springer, p. 248

Ulvestad J.S., Wilson A.S., 1984, ApJ 278, 544

Ulvestad J.S., Wilson A.S., 1984, ApJ 285, 439

Ulvestad J.S., Wilson A.S., 1989, ApJ 343, 659

Ulvestad J.S., Roy A., Colbert J.M., Wilson A.S., 1998, ApJ 496, 196

Wehrle A.E., Morris M., 1988, AJ 95, 1689

Whittle M., Pedlar A., Meurs E.J.A., Unger S.W., Axon D.J., Ward M.J., 1988, ApJ 326, 125

Whittle M., 1989, in Extranuclear Activity in Galaxies, Meurs E.J.A., Fosbury R.A.E. (eds.) ESO Conf Proc. 32, 199

Wilson A.S., Ulvestad J.S., 1983, ApJ 275, 8

Wilson A.S., Ward M.J., Haniff C.A., 1988, ApJ 334, 121

Wilson A.S., Ulvestad J.S., 1987, ApJ 319, 105

Wilson A.S., Tsvetanov Z., 1994, AJ 107, 1227

Wilson A.S., 1991, in "The interpretation of Modern Synthesis Observations of Spiral Galaxies", Duric N., Crane P.C. (eds.) ASP Conf. Ser. 18, 227 